\title{
Three Modern Roles for Logic in Al
}

\author{
Adnan Darwiche \\ Computer Science Department \\ University of California, Los Angeles \\ darwiche@cs.ucla.edu
}

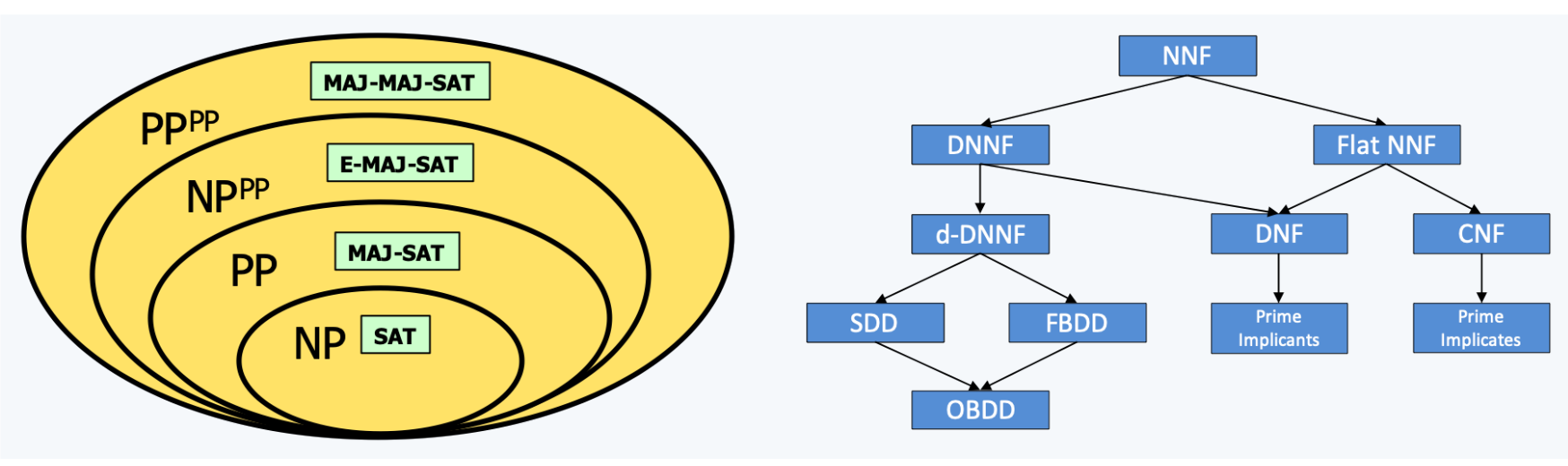

Figure 1: Tractable Boolean circuits as a basis for computation.

\begin{abstract}
We consider three modern roles for logic in artificial intelligence, which are based on the theory of tractable Boolean circuits: (1) logic as a basis for computation, (2) logic for learning from a combination of data and knowledge, and (3) logic for reasoning about the behavior of machine learning systems.
\end{abstract}

\section{CCS CONCEPTS}

- Computing methodologies $\rightarrow$ Learning in probabilistic graphical models; Logical and relational learning; • Theory of computation $\rightarrow$ Automated reasoning; Complexity classes; Problems, reductions and completeness.

\section{KEYWORDS}

tractable circuits, knowledge compilation, explainable AI

\section{ACM Reference Format:}

Adnan Darwiche. 2020. Three Modern Roles for Logic in AI. In Proceedings of the 39th ACM SIGMOD-SIGACT-SIGAI Symposium on Principles of Database Systems (PODS'20), June 14-19, 2020, Portland, OR, USA. ACM, New York, NY, USA, 15 pages. https://doi.org/10.1145/3375395.3389131

\section{INTRODUCTION}

Logic has played a fundamental role in artificial intelligence since the field was incepted [52]. This role has been mostly in the area of knowledge representation and reasoning, where logic is used to

Permission to make digital or hard copies of part or all of this work for personal or classroom use is granted without fee provided that copies are not made or distributed for profit or commercial advantage and that copies bear this notice and the full citation on the first page. Copyrights for third-party components of this work must be honored.

For all other uses, contact the owner/author(s).

PODS'20, June 14-19, 2020, Portland, OR, USA

(c) 2020 Copyright held by the owner/author(s).

ACM ISBN 978-1-4503-7108-7/20/06.

https://doi.org/10.1145/3375395.3389131 represent categorical knowledge and then draw conclusions based on deduction and other more advanced forms of reasoning. Starting with [59], logic also formed the basis for drawing conclusions from a mixture of categorial and probabilistic knowledge.

In this paper, we review three modern roles for propositional logic in artificial intelligence, which are based on the theory of tractable Boolean circuits. This theory, which matured considerably during the last two decades, is based on Boolean circuits in Negation Normal Form (NNF) form. NNF circuits are not tractable, but they become tractable once we impose certain properties on them [34]. Over the last two decades, this class of circuits has been studied systematically across three dimensions. The first dimension concerns a synthesis of NNF circuits that have varying degrees of tractability (the polytime queries they support). The second dimension concerns the relative succinctness of different classes of tractable NNF circuits (the optimal size circuits can attain). The third dimension concerns the development of algorithms for compiling Boolean formula into tractable NNF circuits.

The first modern role for logic we consider is in using tractable circuits as a basis for computation, where we show how problems in the complexity classes NP, PP, NPP and PP ${ }^{\mathrm{PP}}$ can be solved by compiling Boolean formula into corresponding tractable circuits. These are rich complexity classes, which include some commonly utilized problems from probabilistic reasoning and machine learning. We discuss this first role in two steps. In Section 2, we discuss the prototypical problems that are complete for these complexity classes, which are all problems on Boolean formula. We also discuss problems from probabilistic reasoning which are complete for these classes and their reduction to prototypical problems. In Section 3, we introduce the theory of tractable circuits with exposure to circuit types that can be used to efficiently solve problems in these complexity classes (if compiled successfully). 
The second role for logic we shall consider is in learning from a combination of data and symbolic knowledge. We show again that this task can be reduced to a process of compiling, then reasoning with, tractable circuits. This is discussed in Section 4, where we also introduce and employ a class of tractable probabilistic circuits that are based on tractable Boolean circuits.

The third role for logic that we consider is in meta-reasoning, where we employ it to reason about the behavior of machine learning systems. In this role, some common machine learning classifiers are compiled intro tractable circuits that have the same input-output behavior. Queries pertaining to the explainability and robustness of decisions can then be answered efficiently, while also allowing one to formally prove global properties of the underlying machine learning classifiers. This role is discussed in Section 5.

The paper concludes in Section 6 with a perspective on research directions that can mostly benefit the roles we shall discuss, and a perspective on how recent developments in AI have triggered some transitions that remain widely unnoticed.

\section{LOGIC FOR COMPUTATION}

The first role we shall consider for logic is that of systematically solving problems in the complexity classes NP, PP, NPPP and PP PP, which are related in the following way:

$$
\mathrm{NP} \subseteq \mathrm{PP} \subseteq N \mathrm{NP}^{\mathrm{PP}} \subseteq \mathrm{PP} P
$$

The prototypical problems for these complexity classes all correspond to questions on Boolean formula. Moreover, these classes include problems that are commonly used in the areas of probabilistic reasoning and machine learning. While there is a long tradition of developing dedicated algorithms when tackling problems in these complexity classes, it is now common to solve such problems by reducing them to their Boolean counterparts, especially for problems with no tradition of dedicated algorithms.

Let us first consider four common problems from probabilistic reasoning and machine learning that are complete for the classes NP, PP, NPPP and PPPP. These problems can all be stated on probability distributions specified using Bayesian networks [27, 45, 57, 65]. These networks are directed acyclic graphs with nodes representing (discrete) variables. Figure 2 depicts a Bayesian network with five variables SEX, c, T1, T2 and AGREE. The structure of a Bayesian network encodes conditional independence relations among its variables. Each network variable is associated with a set of distributions that are conditioned on the states of its parents (not shown in Figure 2). These conditional probabilities and the conditional independences encoded by the network structure are satisfied by exactly one probability distribution over the network variables. There are four question on this distribution whose decision versions are complete for the complexity classes NP, PP, $\mathrm{NP}^{\mathrm{PP}}$ and PPP . Before we discuss these problems, we settle some notation first.

Upper case letters (e.g., $X$ ) will denote variables and lower case letters (e.g., $x$ ) will denote their instantiations. That is, $x$ is a literal specifying a value for variable $X$. Bold upper case letters (e.g., $\mathrm{X}$ ) will denote sets of variables and bold lower case letters (e.g., $\mathbf{x}$ ) will denote their instantiations. We liberally treat an instantiation of a variable set as conjunction of its corresponding literals.
The four problems we shall consider on a Bayesian network with variables $\mathrm{X}$ and distribution $\operatorname{Pr}(\mathrm{X})$ are MPE, MAR, MAP and SDP. The MPE problem finds an instantiation $\mathbf{x}$ of the network variables that has a maximal probability $\operatorname{Pr}(\mathbf{x})$. This is depicted in Figure 2 which illustrates the result of an MPE computation. The decision version of this problem, D-MPE, asks whether there is a variable instantiation whose probability is greater than a given $k$. The decision problem D-MPE is complete for the class NP [86].

We next have MAR which computes the probability of some value $x$ for a variable $X$. Figure 2 depicts these probabilities for each variable/value pair. The decision version, D-MAR, asks whether $\operatorname{Pr}(x)$ is greater than a given $k$. D-MAR is complete for the class $\mathrm{PP}$ [72]. MAR is perhaps the most commonly used query on Bayesian networks and similar probabilistic graphical models.

The next two problems are stated with respect to a subset of network variables $\mathrm{Y} \subseteq \mathrm{X}$. The problem MAP finds an instantiation $\mathrm{y}$ that has a maximal probability. For example, we may wish to find a most probable instantiation of variables SEx and c in Figure 2. The decision version of this problem, D-MAP, asks whether there is an instantiation $\mathrm{y}$ whose probability is greater than a given $k$. $\mathrm{D}-\mathrm{MAP}$ is complete for the class NPP ${ }^{\mathrm{PP}}\left[64 .^{1}\right.$

Suppose now that we are making a decision based on whether $\operatorname{Pr}(x) \geq T$ for some variable value $x$ and threshold $T$. The SDP problem finds the probability that this decision will stick after having observed the state of variables Y. For example, we may want to operate on a patient if the probability of condition $\mathrm{C}$ in Figure 2 is greater than $90 \%$ (the decision is currently negative). The SDP (same-decision probability) can be used to compute the probability that this decision will stick after having obtained the results of tests $\mathrm{T} 1$ and $\mathrm{T} 2$ [31]. The SDP computes an expectation. Its decision version, D-SDP, asks whether this probability is greater than a given $k$ and is complete for the class $\mathrm{PP}^{\mathrm{PP}}$ [18]. This query was used to assess the value of information with applications to reasoning about features in Bayesian network classifiers; see, e.g., [11, 19, 20].

There is a tradition of solving the above problems using dedicated algorithms; see, e.g., the treatments in [27, 45, 57, 65]. Today these problems are also being commonly solved using reductions to the prototypical problems of the corresponding complexity classes, which are defined over Boolean formula as mentioned earlier. This is particularly the case for the MAR problem and for distributions that are specified by representations that go beyond Bayesian networks; see, e.g., $[35,47,50]$. These reduction-based approaches are the state of the art on certain problems; for example, when the Bayesian network has an abundance of $0 / 1$ probabilities or context-specific independence [32]. ${ }^{2}$

We next discuss the prototypical problems of complexity classes $\mathrm{NP}, \mathrm{PP}, \mathrm{NP} \mathrm{PP}^{\mathrm{PP}}$ and PPP before we illustrate in Section 2.2 the core technique used in reductions to these problems. We then follow in Section 3 by showing how these prototypical problems can be solved systematically by compiling Boolean formula into Boolean circuits with varying degrees of tractability.

\footnotetext{
${ }^{1}$ Some treatments in the literature use MAP and partial MAP when referring to MPE and MAP, respectively. Our treatment follows the original terminology used in [65]. ${ }^{2}$ Context-specific independence refers to independence relations among network variables which are implied by the specific probabilities that quantify the network and, hence, cannot be detected by only examining the network structure [4].
} 

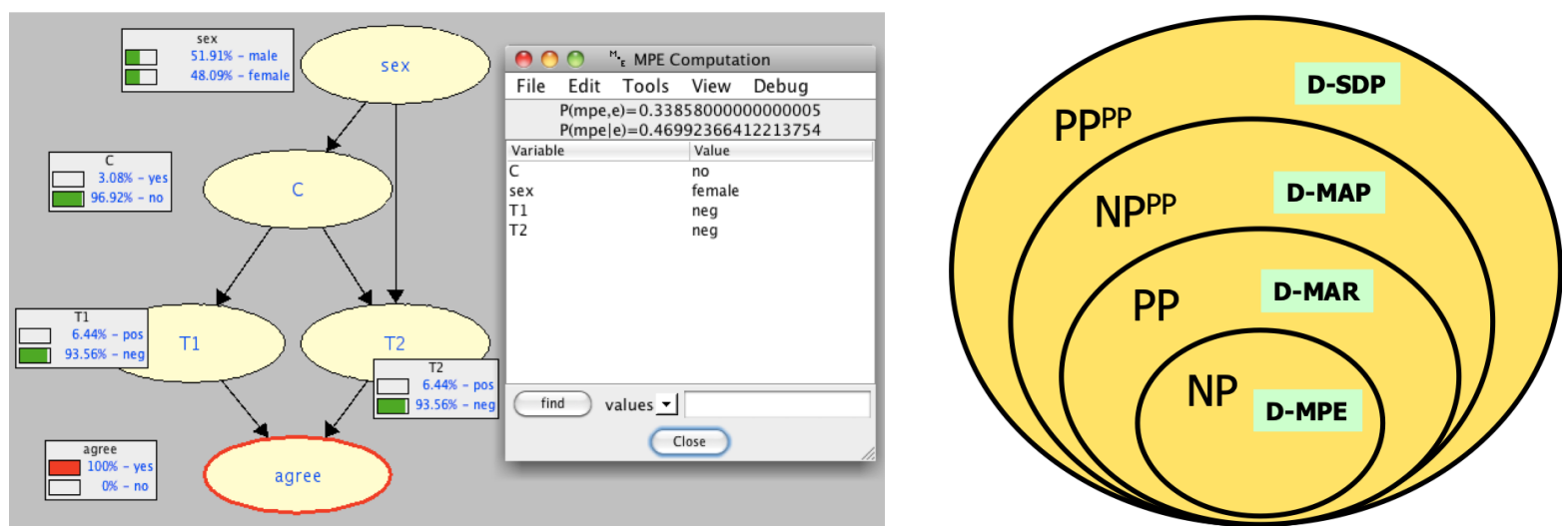

Figure 2: Left: A Bayesian network with an illustration of the MPE query. The network concerns a medical condition c and two tests $\mathrm{T} 1$ and $\mathrm{T} 2$ that can be used to detect the condition. The variable AGREE indicates whether the two test results are in agreement. Right: Decision problems on Bayesian networks that are complete for the classes $N P, P P, N P^{P P}$ and $P P^{P P}$.

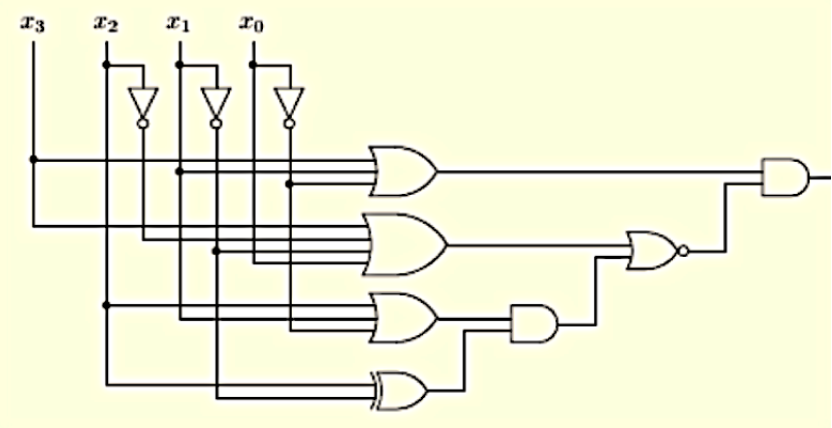

Figure 3: A Boolean circuit to illustrate the problems SAT, MAJSAT, E-MAJSAT and MAJMAJSAT.

\subsection{Prototypical Problems}

NP is the class of decision problems that can be solved by a nondeterministic polynomial-time Turing machine. PP is the class of decision problems that can be solved by a non-deterministic polynomial-time Turing machine, which has more accepting than rejecting paths. NPPP and $\mathrm{PP}^{\mathrm{PP}}$ are the corresponding classes assuming the corresponding Turing machine has access to a PP oracle.

The prototypical problems for these classes are relatively simple and are usually defined on Boolean formula in Conjunctive Normal Form (CNF). We will however illustrate them on Boolean circuits to make a better connection to the discussion in Section 3 .

Consider a Boolean circuit $\Delta$ which has input variables $\mathrm{X}$ and let $\Delta(\mathbf{x})$ be the output of this circuit given input $\mathbf{x}$; see Figure 3 . The following decision problems are respectively complete and prototypical for the complexity classes NP, PP, NPPP and PPPP.

-SAT asks if there is a circuit input $\mathbf{x}$ such that $\Delta(\mathbf{x})=1$.

- MAJSAT asks if the majority of circuit inputs $\mathbf{x}$ are such that $\Delta(\mathbf{x})=1$.

The next two problems require that we partition the circuit input variables $\mathrm{X}$ into two sets $\mathrm{Y}$ and $\mathrm{Z}$.
-E-MAJSAT asks if there is a circuit input $\mathrm{y}$ such that $\Delta(\mathrm{y}, \mathrm{z})=1$ for the majority of circuit inputs $\mathrm{z}$.

-MAJMAJSAT asks if the majority of circuit inputs $y$ are such that $\Delta(\mathbf{y}, \mathbf{z})=1$ for the majority of circuit inputs $\mathbf{z}$.

There are two functional versions of MAJSAT which have been receiving increased attention recently. The first is \#SAT which asks for the number of circuit inputs $\mathbf{x}$ such that $\Delta(\mathbf{x})=1$. Algorithms that solve this problem are known as model counters. ${ }^{3}$ The more general functional version of MAJSAT and the one typically used in practical reductions is called weighted model counting, WMC. In this variant, each circuit input $x$ for variable $X$ is given a weight $W(x)$. A circuit input $\mathbf{x}=x_{1}, \ldots, x_{n}$ is then assigned the weight $W(\mathbf{x})=W\left(x_{1}\right) \ldots W\left(x_{n}\right)$. Instead of counting the number of inputs $\mathbf{x}$ such that $\Delta(\mathbf{x})=1$, weighted model counting adds up the weights of such inputs. That is, WMC computes $\sum_{\mathbf{x}} W(\mathbf{x})$ for all circuit inputs $\mathbf{x}$ such that $\Delta(\mathbf{x})=1$.

\subsection{The Core Reduction}

As mentioned earlier, the decision problems SAT, MAJSAT, E-MAJSAT and MAJMAJSAT on Boolean formula are complete and prototypical for the complexity classes NP, PP, NPPP and PPP . The decision problems D-MPE, D-MAR, D-MAP and D-SDP on Bayesian networks are also complete for these complexity classes, respectively. Practical reductions of the latter problems to the former ones have been developed over the last two decades; see [27, Chapter 11] for a detailed treatment. Reductions have also been proposed from the functional problem MAR to WMC, which are of most practical significance. We will next discuss the first such reduction [24] since it is relatively simple yet gives the essence of how one can reduce problems that appear in probabilistic reasoning and machine learning to problems on Boolean formula and circuits. In Section 3, we will further show how these problems (of numeric nature) can be competitively solved using purely symbolic manipulations.

\footnotetext{
${ }^{3}$ Some of the popular or traditional model counters are c2D [26], MINI-C2D [63], D4 [46], CACHE [73], SHARP-SAT [88], SDD [12] and DSHARP [56]. Many of these systems can also compute weighted model counts.
} 


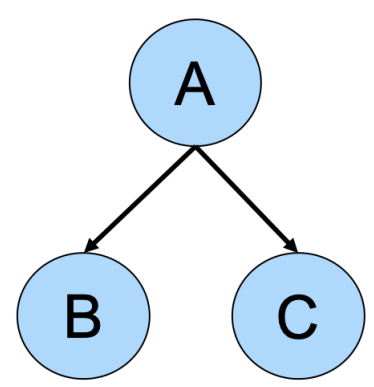

\begin{tabular}{|l|l|l|l|}
\hline $\mathrm{A}$ & $\mathrm{B}$ & $\mathrm{C}$ & $\operatorname{Pr}()$. \\
\hline $\mathrm{T}$ & $\mathrm{T}$ & $\mathrm{T}$ & $\theta_{A} \theta_{B \mid A} \theta_{C \mid A}$ \\
\hline $\mathrm{T}$ & $\mathrm{T}$ & $\mathrm{F}$ & $\theta_{A} \theta_{B \mid A} \theta_{\neg C \mid A}$ \\
\hline $\mathrm{T}$ & $\mathrm{F}$ & $\mathrm{T}$ & $\theta_{A} \theta_{\neg B \mid A} \theta_{C \mid A}$ \\
\hline $\mathrm{T}$ & $\mathrm{F}$ & $\mathrm{F}$ & $\theta_{A} \theta_{\neg B \mid A} \theta_{\neg C \mid A}$ \\
\hline $\mathrm{F}$ & $\mathrm{T}$ & $\mathrm{T}$ & $\theta_{\neg A} \theta_{B \mid \neg A} \theta_{C \mid \neg A}$ \\
\hline $\mathrm{F}$ & $\mathrm{T}$ & $\mathrm{F}$ & $\theta_{\neg A} \theta_{B \mid \neg A} \theta_{\neg C \mid \neg A}$ \\
\hline $\mathrm{F}$ & $\mathrm{F}$ & $\mathrm{T}$ & $\theta_{\neg A} \theta_{\neg B \mid \neg A} \theta_{C \mid \neg A}$ \\
\hline $\mathrm{F}$ & $\mathrm{F}$ & $\mathrm{F}$ & $\theta_{\neg A} \theta_{\neg B \mid \neg A} \theta_{\neg C \mid \neg A}$ \\
\hline
\end{tabular}

Figure 4: A Bayesian network and its distribution.

Consider the Bayesian network in Figure 4, which has three binary variables $A, B$ and $C$. Variable $A$ has one distribution $\left(\theta_{A}, \theta_{\neg A}\right)$. Variable $B$ has two distributions, which are conditioned on the state of its parent $A$ : $\left(\theta_{B \mid A}, \theta_{\neg B \mid A}\right)$ and $\left(\theta_{B \mid \neg A}, \theta_{\neg B \mid \neg A}\right)$. Variable $C$ also has two similar distributions: $\left(\theta_{C \mid A}, \theta_{\neg C \mid A}\right)$ and $\left(\theta_{C \mid \neg A}, \theta_{\neg C \mid \neg A}\right)$. We will refer to the probabilities $\theta$ as network parameters. The Bayesian network in Figure 4 has ten parameters.

This Bayesian network induces the distribution depicted in Figure 4 , where the probability of each variable instantiation is simply the product of network parameters that are compatible with that instantiation; see [27, Chapter 3] for a discussion of the syntax and semantics of Bayesian networks. We will next show how one can efficiently construct a Boolean formula $\Delta$ from a Bayesian network, allowing one to compute marginal probabilities on the Bayesian network by performing weighted model counting on formula $\Delta$.

The main insight is to introduce a Boolean variable $P$ for each network parameter $\theta$, which is meant to capture the presence or absence of parameter $\theta$ given an instantiation of the network variables (i.e., a row of the table in Figure 4). For the network in Figure 4 , this leads to introducing ten Boolean variables: $P_{A}, P_{\neg A}$, $P_{B \mid A}, \ldots, P_{\neg C \mid \neg A}$. In the second row of Figure 4 , which corresponds to variable instantiation $A, B, \neg C$, parameters $\theta_{A}, \theta_{B \mid A}$ and $\theta_{\neg C \mid A}$ are present and the other seven parameters are absent.

We can capture such presence/absence by adding one expression to the Boolean formula $\Delta$ for each network parameter. For example, the parameters associated with variable $A$ introduce the following expressions: $A \Longleftrightarrow P_{A}$ and $\neg A \Longleftrightarrow \neg P_{\neg A}$. Similarly, the parameters of variable $B$ introduce the expressions $A \wedge B \Longleftrightarrow$ $P_{B \mid A}, A \wedge \neg B \Longleftrightarrow P_{\neg B \mid A}, \neg A \wedge B \Longleftrightarrow P_{B \mid \neg A}$ and $\neg A \wedge \neg B \Longleftrightarrow$ $P_{\neg B \mid \neg A}$. The parameters of variable $C$ introduce similar expressions.

The resulting Boolean formula $\Delta$ will have exactly eight models, which correspond to the network instantiations. The following is one of these models which correspond to instantiation $A, B, \neg C$ :

$$
\begin{array}{r}
A B \neg C P_{A} P_{B \mid A} P_{\neg C \mid A} \\
\neg P_{\neg A} \quad \neg P_{\neg B \mid A} \neg P_{B \mid \neg A} \neg P_{\neg B \mid \neg A} \quad \neg P_{C \mid A} \neg P_{C \mid \neg A} \neg P_{\neg C \mid \neg A} .
\end{array}
$$

In this model, all parameters associated with instantiation $A, B, \neg C$ appear positively (present) while others appear negatively (absent).

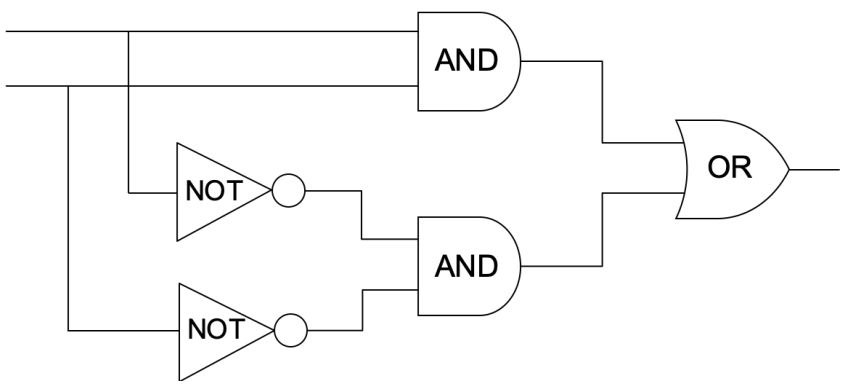

Figure 5: Negation Normal Form (NNF) circuit.

The last step is to assign weights to the values of variables (literals). For network variables, all literals get a weight of 1 ; for example, $W(A)=1$ and $W(\neg A)=1$. The negative literals of parameter variables also get a weight of 1 ; for example, $W\left(\neg P_{A}\right)=1$ and $W\left(\neg P_{\neg C \mid A}\right)=1$. Finally, positive literals of network parameters get weights equal to these parameters; for example, $W\left(P_{A}\right)=\theta_{A}$ and $W\left(P_{\neg C \mid A}\right)=\theta_{\neg C \mid A}$. The weight of expression (1) is then $\theta_{A} \theta_{B \mid A} \theta_{\neg C \mid A}$, which is precisely the probability of network instantiation $A, B, \neg C$. We can now compute the probability of any Boolean expression $\alpha$ by simply computing the weighted model count of $\Delta \wedge \alpha$, which completes the reduction of MAR to WMC.

Another reduction was proposed in [73] which is suited towards Bayesian networks that have variables with large cardinalities. More refined reductions have also been proposed which can capture certain properties of network parameters such as $0 / 1$ parameters and context-specific independence (can be critical for the efficient computation of weighted model counts on the resulting Boolean formula). A detailed treatment of reduction techniques and various practical tradeoffs can be found in [10] and [27, Chapter 13]. ${ }^{4}$

\section{TRACTABLE CIRCUITS}

We now turn to a systematic approach for solving prototypical problems in the classes NP, PP, NPPP and PPP , which is based on compiling Boolean formula into tractable Boolean circuits. The circuits we shall compile into are in Negation Normal Form (NNF) as depicted in Figure 5. These circuits have three types of gates: and-gates, or-gates and inverters, except that inverters can only feed from the circuit variables. Any circuit with these types of gates can be converted to an NNF circuit while at most doubling its size.

NNF circuits are not tractable. However, by imposing certain properties on them we can attain different degrees of tractability. The results we shall review next are part of the literature on knowledge compilation, an area that has been under development for a few decades, see, e.g., $[8,51,74]$, except that it took a different turn since [34]; see also [29]. Earlier work on knowledge compilation focused on flat NNF circuits, which include subsets of Conjunctive Normal Form (CNF) and Disjunctive Normal Form (DNF) such as prime implicates, Horn clauses and prime implicants. Later, however, the focus shifted towards deep NNF circuits with no restriction on the number of circuit layers. A comprehensive treatment was initially given in [34], in which some tractable NNF circuits were studied across the two dimensions of tractability and

\footnotetext{
${ }^{4}$ ACE implements some of these reductions: http://reasoning.cs.ucla.edu/ace/
} 


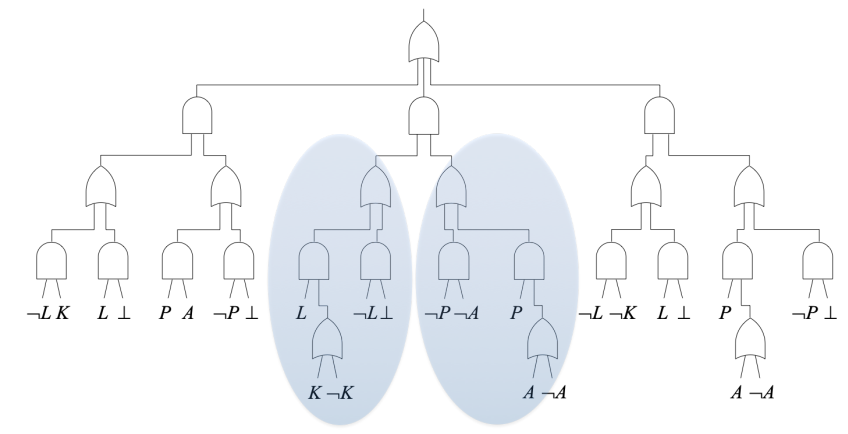

Figure 6: Illustrating the decomposability property of NNF circuits. The illustration does not tie shared inputs of the circuit for clarity of exposition.

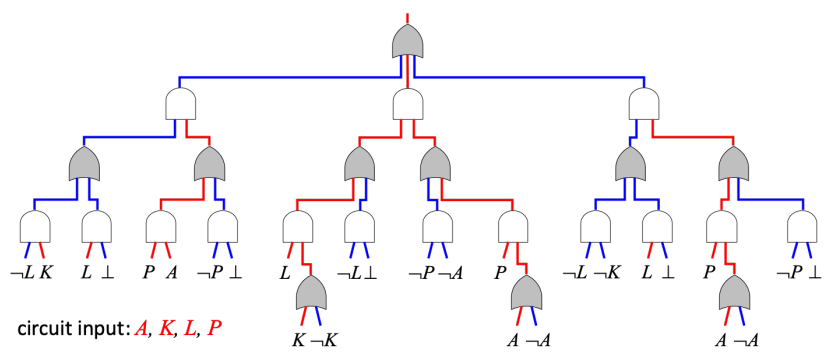

Figure 7: Illustrating the determinism property of NNF circuits. Red wires are high and blue ones are low.

succinctness. As we increase the strength of properties imposed on NNF circuits, their tractability increases by allowing more queries to be performed in polytime. This, however, typically comes at the expense of succinctness as the size of circuits gets larger.

One of the simplest properties that turn NNF circuits into tractable ones is decomposability [22]. According to this property, subcircuits feeding into an and-gate cannot share circuit variables. Figure 6 illustrates this property by highlighting the two subcircuits (in blue) feeding into an and-gate. The subcircuit on the left feeds from circuit variables $K$ and $L$, while the one on the right feeds from circuit variables $A$ and $P$. NNF circuits that satisfy the decomposability property are known as Decomposable NNF (DNNF) circuits. The satisfiability of DNNF circuits can be decided in time linear in the circuit size [22]. Hence, enforcing decomposability is sufficient to unlock the complexity class NP.

The next property we consider is determinism [23], which applies to or-gates in an NNF circuit. According to this property, at most one input for an or-gate must be high under any circuit input. Figure 7 illustrates this property when all circuit variables $A, K, L, P$ are high. Examining the or-gates in this circuit, under this circuit input, one sees that each or-gate has either one high input or no high inputs. This property corresponds to mutual exclusiveness when the or-gate is viewed as a disjunction of its inputs. MAJSAT can be decided in polytime on NNF circuits that are both decomposable and deterministic. These circuits are called d-DNNF circuits. If they are also smooth [25], a property that can be enforced in quadratic time, d-DNNF circuits allow one to perform weighted model counting

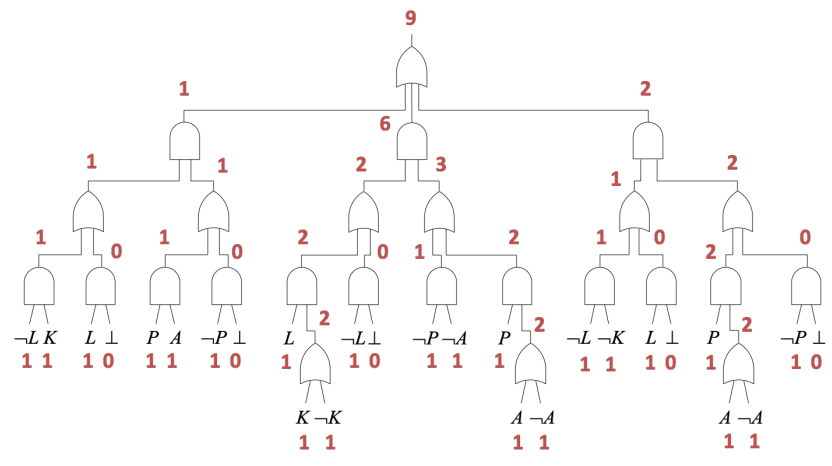

Figure 8: Model counting in linear time on d-DNNF circuits.

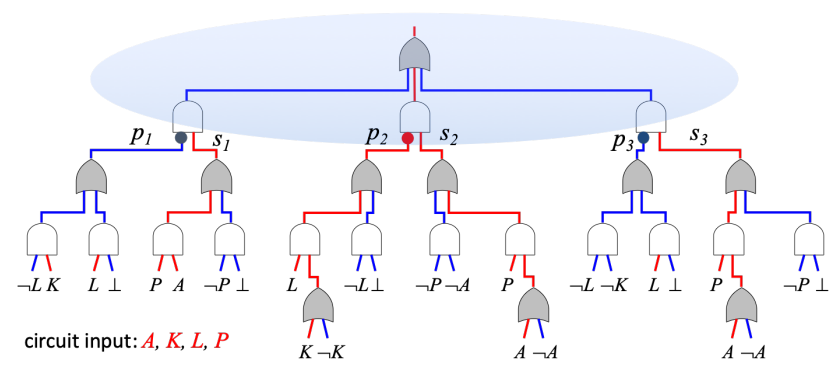

Figure 9: Illustrating the sentential decision property of NNF circuits. Red wires are high and blue ones are low.

(WMC) in linear time. ${ }^{5}$ The combination of decomposability and determinism therefore unlocks the complexity class PP.

Smoothness requires that all subcircuits feeding into an or-gate mention the same circuit variables. For example, in Figure 7, three subcircuits feed into the top or-gate. Each of these subcircuits mentions the same set of circuit variables: $A, K, L, P$. Enforcing smoothness can introduce trivial gates into the circuit such as the bottom three or-gates in Figure 7 and can sometimes be done quite efficiently [85]. An example of model counting using a d-DNNF circuit is depicted in Figure 8. Every circuit literal, whether a positive literal such as $A$ or a negative literal such as $\neg A$, is assigned the value 1 . Constant inputs $T$ and $\perp$ are assigned the values 1 and 0 . We then propagate these numbers upwards, multiplying numbers assigned to the inputs of an and-gate and summing numbers assigned to the inputs of an or-gate. The number we obtain for the circuit output is the model count. In this example, the circuit has 9 satisfying inputs out of 16 possible ones. To perform weighted model counting, we simply assign a weight to a literal instead of the value $1-$ model counting (\#SAT) is a special case of weighted model counting (WMC) when the weight of each literal is 1 .

There are stronger versions of decomposability and determinism which give rise to additional, tractable NNF circuits. Structured decomposability is stated with respect to a binary tree whose leaves are in one-to-one correspondence with the circuit variables [66]. Such a tree is depicted in Figure 10(a) and is known as a vtree.

\footnotetext{
${ }^{5}$ One can actually compute all marginal, weighted model counts in linear time [23].
} 


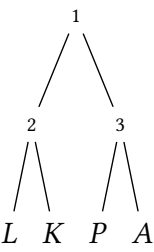

(a) vtree

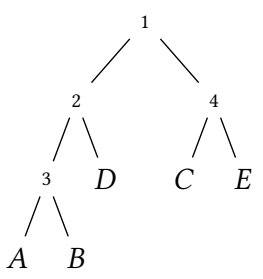

(b) constrained $C E \mid A B D$

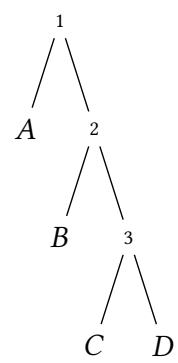

(c) right-linear
Figure 10: A constrained vtree for $\mathrm{X} \mid \mathrm{Y}$ is a vtree over variables $\mathrm{X} \cup \mathrm{Y}$ that contains a node $u$ with following properties: (1) $u$ can be reached from the vtree root by following right children only and (2) the variables of $u$ are precisely X. A rightlinear vtree is one in which the left child of every internal node is a leaf.

Structured decomposability requires that each and-gate has two inputs $i_{1}$ and $i_{2}$ and correspond to a vtree node $v$ such that the variables of subcircuits feeding into $i_{1}$ and $i_{2}$ are in the left and right subtrees of vtree node $v$. The DNNF circuit in Figure 6 is structured according to the vtree in Figure 10(a). For example, the and-gate highlighted in Figure 6 respects vtree node $v=1$ in Figure 10(a). Two structured DNNF circuits can be conjoined in polytime to yield a structured DNNF, which cannot be done on DNNF circuits under standard complexity assumptions [34].

Structured decomposability, together with a stronger version of determinism, yields a class of NNF circuits known as Sentential Decision Diagrams (SDDs) [28]. To illustrate this stronger version of determinism, consider Figure 9 and the highlighted circuit fragment. The fragment corresponds to the Boolean expression $\left(p_{1} \wedge s_{1}\right) \vee$ $\left(p_{2} \wedge s_{2}\right) \vee\left(p_{3} \wedge s_{3}\right)$, where each $p_{i}$ is called a prime and each $s_{i}$ is called a $s u b$ (primes and subs correspond to subcircuits). Under any circuit input, precisely one prime will be high. In Figure 9, under the given circuit input, prime $p_{2}$ is high while primes $p_{1}$ and $p_{3}$ are low. This means that this circuit fragment, which acts as a multiplexer, is actually passing the value of sub $s_{2}$ while suppressing the value of subs $s_{1}$ and $s_{3}$. As a result, the or-gate in this circuit fragment is guaranteed to be deterministic: at most one input of the or-gate will be high under any circuit input. SDD circuits result from recursively applying this multiplexer construct, which implies determinism, to a given vtree (the SDD circuit in Figure 9 is structured with respect to the vtree in Figure 10(a)). See [28] for the formal definitions of SDD circuits and the underlying stronger version of determinism. ${ }^{6}$

SDDs support polytime conjunction and disjunction. That is, given two SDDs $\alpha$ and $\beta$, there is a polytime algorithm to construct another SDD $\gamma$ that represents $\alpha \wedge \beta$ or $\alpha \vee \beta .^{7}$ SDDs can also be negated in linear time. The size of an SDD can be very sensitive to the underlying vtree, ranging from linear to exponential in some

\footnotetext{
${ }^{6}$ The vtree of an SDD is ordered: the distinction between left and right children matters ${ }^{7}$ If $s$ and $t$ are the sizes of input SDDs, then conjoining or disjoining the SDDs takes $O(s \cdot t)$ time, although the resulting SDD may not be compressed [89]. Compression is a property that ensures the canonicity of an SDD for a given vtree [28].
}
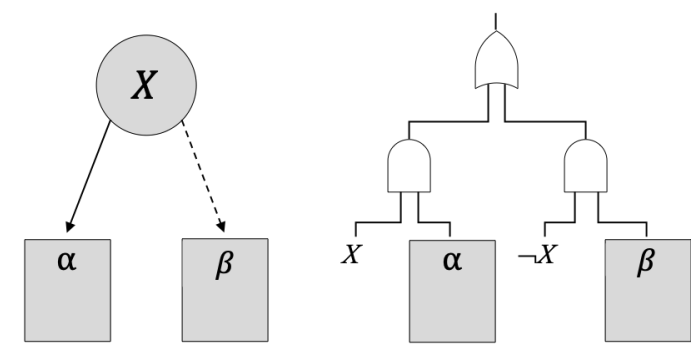

Figure 11: OBDD fragment and corresponding NNF circuit.

cases $[12,93]$. Recall that E-MAJSAT and MAJMAJSAT, the prototypical problems for the complexity classes $\mathrm{NP}^{\mathrm{PP}}$ and $\mathrm{PP} P \mathrm{PP}$, are stated with respect to a split of variables in the Boolean forumla. If the vtree is constrained according to this split, then these problems can be solved in linear time on the corresponding SDD [61]. ${ }^{8}$ Figure 10(b) illustrates the concept of a constrained vtree.

SDDs subsume the well known Ordered Binary Decision Diagrams (OBDDs) and are exponentially more succinct than OBDDs [5]; see also [3]. An OBDD is an ordered decision graph: the variables on every path from the root to a leaf ( 0 or 1$)$ respect a given variable order $[7,55,90]$; see Figure 25 . An OBDD node and its corresponding NNF fragment are depicted in Figure 11. As the figure shows, this fragment is also a multiplexer as in SDDs, except that we have precisely two primes which correspond to a variable and its negation. When an SDD is structured with respect to a right-linear vtree, the result is an OBDD; see Figure 10(c). SDDs and OBDDs can also be contrasted based on how they make decisions. An OBDD makes a decision based on the state of a binary variable and hence its decisions are always binary. An SDD makes decisions based on sentences (primes) so the corresponding number of decisions (subs) is not restricted to being binary.

The compilation of Boolean formula into tractable NNF circuits is done by systems known as knowledge compilers. Examples include C2D ${ }^{9}$ [26], CUDD ${ }^{10}$, MINI-C2D ${ }^{11}[62,63], \mathrm{D}^{12}$ [46], DSHARP ${ }^{13}$ [56] and the SDD library [12]. ${ }^{14}$ See also http://beyondnp.org/pages/ solvers/knowledge-compilers/. A connection was made between model counters and knowledge compilers in [38], showing how model counters can be turned into knowledge compilers by keeping a trace of the exhaustive search they conduct on a Boolean formula. The DSHARP compiler for d-DNNF [56] was the result of keeping a trace of the SHARP-SAT model counter [88]. While dedicated SAT solvers remain the state of the art for solving SAT, the state of the art for (weighted) model counting are either knowledge compilers or model counters whose traces are (subsets) of d-DNNF circuits.

\footnotetext{
${ }^{8} \mathrm{~A}$ weaker condition exists for the class $\mathrm{NP} \mathrm{PP}^{\mathrm{PP}}$ but is stated on another circuit type known as Decision-DNNF [38], which we did not introduce in this paper; see [37, 67]. ${ }^{9}$ http://reasoning.cs.ucla.edu/c2d

${ }^{10} \mathrm{https} / / /$ davidkebo.com/cudd

${ }^{11} \mathrm{http}: / /$ reasoning.cs.ucla.edu/minic2d

${ }^{12} \mathrm{http}: / /$ www.cril.univ-artois.fr/kc/d4

${ }^{13} \mathrm{https} / / /$ bitbucket.org/haz/dsharp

${ }^{14} \mathrm{http}: / /$ reasoning.cs.ucla.edu/sdd (open source). A Python wrapper of the SDD library is available at https://github.com/wannesm/PySDD and an NNF-to-SDD circuit compiler, based on PySDD, is available open-source at https://github.com/art-ai/nnf2sdd
} 


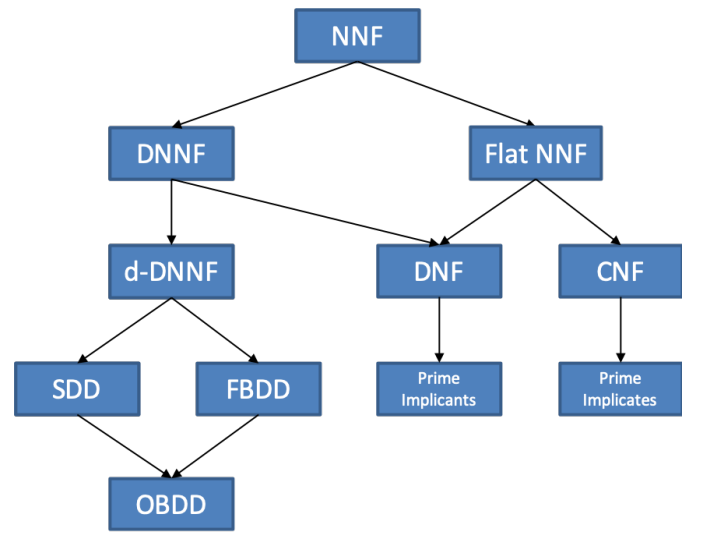

Figure 12: A partial taxonomy of NNF circuits.

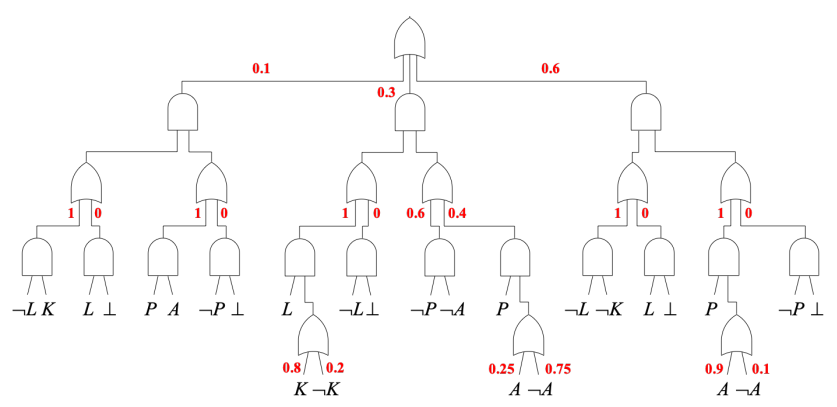

Figure 13: A probabilistic SDD circuit. The probabilities annotating inputs of or-gate are known as PSDD parameters.

Figure 12 depicts a partial taxonomy of NNF circuits, most of which are tractable. A more comprehensive taxonomy was studied in [34] under the term knowledge compilation map. A recent update on this map can be found in [87], which includes recent results on the exponential separations between tractable NNF circuits.

Tractable NNF circuits were connected to, utilized by, and also benefited from other areas of computer science. This includes database theory, where connections were made to the notions of provenance and lineage, e.g., [1, 42]. It also includes communication complexity, which led to sharper separation results between tractable NNF circuits, e.g., [2, 6]. Further applications to probabilistic reasoning and machine learning include the utilization of tractable circuits for uniform sampling [75] and improving deep learning through the injection of symbolic knowledge; see, e.g., [91, 92].

\section{LOGIC FOR LEARNING FROM DATA AND KNOWLEDGE}

The second role we consider for logic is in learning distributions from a combination of data and symbolic knowledge. This role has two dimensions: representational and computational. On the representational side, we use logic to eliminate situations that are impossible according to symbolic knowledge, which can reduce the amount of needed data and increase the robustness of learned

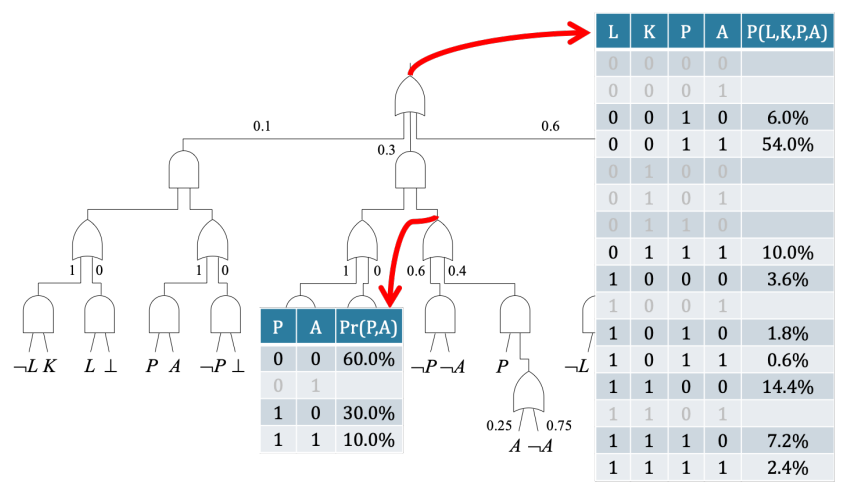

Figure 14: The compositional distributions of a PSDD circuit.

representations. On the computational side, we use logic to factor the space of possible situations into a tractable NNF circuit so we can learn a distribution over the space and reason with it efficiently.

Consider the SDD circuit in Figure 13 which we showed earlier to have 9 satisfying inputs out of 16 . Suppose that our goal is to induce a distribution on this space of satisfying circuit inputs. We can do this by simply assigning a distribution to each or-gate in the circuit as shown in Figure 13: each input of an or-gate is assigned a probability while ensuring that the probabilities assigned to the inputs add up to 1 . These local distributions are independent of one another. Yet, they are guaranteed to induce a normalized distribution over the space of satisfying circuit inputs. The resulting circuit is known as a probabilistic SDD circuit or a PSDD for short [44].

Given a PSDD with variables $\mathrm{X}$, the distribution $\operatorname{Pr}(\mathrm{X})$ it induces can be obtained as follows. To compute the probability of input $\mathbf{x}$ we perform a bottom-up pass on the circuit while assigning a value to each literal and gate output. The value of a literal is just its value in input $\mathbf{x}(1$ or 0$)$. The value of an and-gate is the product of values assigned to its inputs. The value of an or-gate is the weighted sum of values assigned to its inputs (the weights are the probabilities annotating the gate inputs). The value assigned to the circuit output will then be the probability $\operatorname{Pr}(\mathbf{x})$. Figure 14 depicts the result of applying this evaluation process. As the figure shows, the probabilities of satisfying circuit inputs add up to 1 . Moreover, the probability of each unsatisfying input is 0 . The PSDD has a compositional semantics: each or-gate induces a distribution over the variables in the subcircuit rooted at the gate. Figure 14 depicts the distribution induced by an or-gate over variables $P$ and $A$.

To see how PSDDs can be used to learn from a combination of data and symbolic knowledge, consider the example in Figure 15 from [44]. What we have here is a dataset that captures student enrollments in four courses offered by a computer science department. Each row in the dataset represents the number of students who have enrolled in the corresponding courses. Our goal is to learn a distribution from this data and the knowledge we have about the course prerequisites and requirements that is shown in Figure 15.

This symbolic knowledge can be captured by the propositional statement $(P \vee L) \wedge(A \Rightarrow P) \wedge(K \Rightarrow(A \vee L))$. The first step is to compile this knowledge into an SDD circuit. The SDD circuit in 


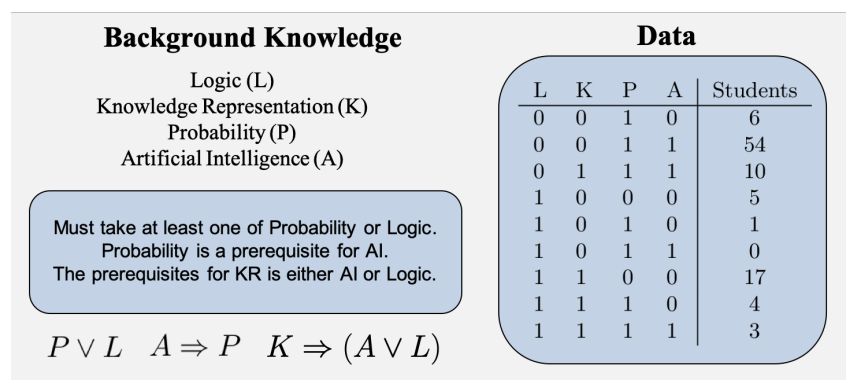

Figure 15: Learning from data and knowledge.

Figure 13 is actually the result of this compilation (using the vtree in Figure 10(a)). This SDD circuit will evaluate to 1 for any course combination that is allowed by the prerequisites and requirements, otherwise it will evaluate to 0 . Our next step is to induce a distribution on the satisfying inputs of this course, that is, the valid course combinations. This can be done by learning a distribution for each or-gate in the SDD circuit, to yield a PSDD circuit.

The data in Figure 15 is complete in the sense that each row specifies precisely whether a course was enrolled into or not by the corresponding number of students. An incomplete dataset in this case would, for example, specify that 30 students took logic, $\mathrm{AI}$ and probability, without specifying the status of enrollment in knowledge representation. If the data is complete, the maximumlikelihood parameters of a PSDD can be learned in time linear in the PSDD size. All we need to do is evaluate the SDD circuit for each example in the dataset, while keeping track of how many times a wire become high; see [44] for details. The parameters shown in Figure 13 were actually learned using this procedure so the distribution they induce is the one guaranteed to maximize the probability of given data (under the chosen vtree). ${ }^{15}$

Both MPE and MAR queries, discussed in Section 2, can be computed in time linear in the PSDD size [44]. Hence, not only can we estimate parameters efficiently under complete data, but we can also reason efficiently with the learned distribution. Finally, the PSDD is a complete and canonical representation of probability distributions. That is, PSDDs can represent any distribution, and there is a unique PSDD for that distribution (under some conditions) [44].

PSDD circuits are based on the stronger versions of decomposability and determinism that underly SDD circuits. Probabilistic circuits that are based on the standard properties of decomposability and determinism are called ACs (Arithmetic Circuits) [25]. Those based on decomposability only were introduced about ten years later and are known as SPNs (Sum-Product Networks) [68] A treatment on the relative tractability and succinctness of these three circuit types can be found in $[13,76]$.

\subsection{Learning With Combinatorial Spaces}

We now discuss another type of symbolic knowledge that arises when learning distributions over combinatorial objects.

Consider Figures 16 and 17 which depicts two common types of combinatorial objects: routes and total orderings. In the case of

\footnotetext{
${ }^{15}$ See https://github.com/art-ai/pypsdd for a package that learns PSDDs and http: //reasoning.cs.ucla.edu/psdd/ for additional tools.
}

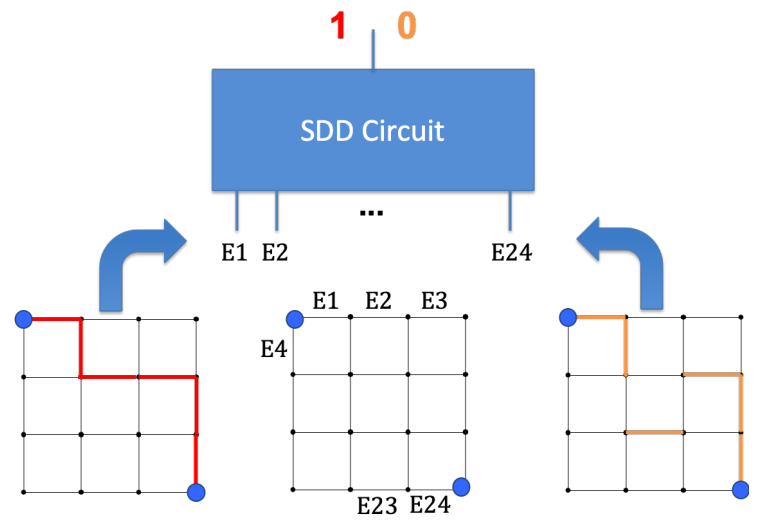

Figure 16: Encoding routes using SDDs. The red variable assignment (left) encoudes a valid route. The orange variable assignment (right) does not encode a valid route as the edges are disconnected.

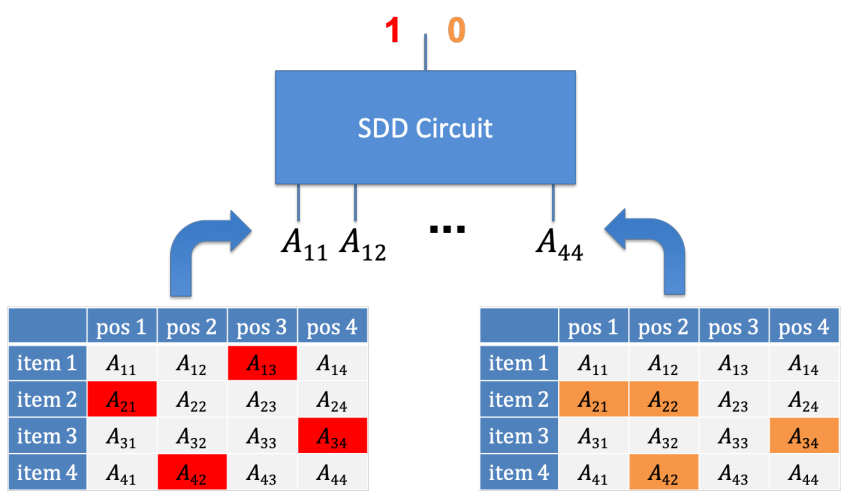

Figure 17: Encoding rankings (total orderings) using SDDs. The red variable assignment (left) encodes a valid ranking. The orange variable assignment (right) does not encode a valid ranking (e.g., item 2 appears in two positions).

routes, we have a map (in this case a grid for simplicity), a source and a destination. Our goal is to learn a distribution over the possible routes from the source to the destination given a dataset of routes, as discussed in [16]. In the case of total orderings (rankings), we have $n$ items and we wish to learn a distribution over the possible rankings of these items, again from a dataset of rankings, as discussed in [17].

It is not uncommon to develop dedicated learning and inference algorithms when dealing with combinatorial objects. For example, a number of dedicated frameworks exist for learning and inference with distributions over rankings; see, e.g., [36, 49, 54]. What we will do, however, is show how learning and inference with combinatorial objects including rankings can be handled systematically using tractable circuits as proposed in [14, 16, 17, 77, 79].

Consider the grid in Figure 16 (more generally, a map is modeled using an undirected graph). We can represent each edge $i$ in the map by a Boolean variable $E_{i}$ and each route as a variable assignment that sets only its corresponding edge variables to true. While every 
route can be represented by a variable assignment (e.g., the red route on the left of Figure 16), some assignments will correspond to invalid routes (e.g., the orange one on the right of Figure 16). One can construct a Boolean formula over edge variables whose satisfying assignments correspond precisely to valid (connected) routes and include additional constraints to keep only, for example, simple routes with no cycles; see $[16,60]$ for how this can be done.

After capturing the space of valid routes using an appropriate Boolean formula, we compile it to an SDD circuit. Circuit inputs that satisfy the SDD will then correspond to the space of valid routes; see Figure 16. A complete dataset in this case will be a multiset of variable assignments, each corresponding to a taken route (obtained, for example, from GPS data). We can then learn PSDD parameters from this dataset and compiled SDD as done in $[14,16]$

Figure 17 contains another example of applying this approach to learning distributions over rankings with $n$ items. In this case, we use $n^{2}$ Boolean variables $A_{i j}$ to encode a ranking, by setting variable $A_{i j}$ to true iff item $i$ is in position $j$. One can also construct a Boolean formula whose satisfying assignments correspond precisely to valid rankings. Compiling the formula to an SDD and then learning PSDD parameters from data allow us to learn a distribution over rankings that can be reasoned about efficiently. This proposal for rankings (and partial rankings, including tiers) was extended in [17], showing competitive results with some dedicated approaches for learning distributions over rankings. It also included an account for learning PSDD parameters from incomplete data and from structured data in which examples are expressed using arbitrary Boolean formula instead of just variable assignments.

A combinatorial probability space is a special case of the more general structured probability space, which is specified by a Boolean formula (i.e., the satisfying assignments need not correspond to combinatorial objects). The contrast is a standard probability space that is defined over all instantiations of a set of variables. We will use the term structured instead of combinatorial in the next section, as adopted in earlier works $[14,16,17,79]$, even as we continue to give examples from combinatorial spaces.

\subsection{Conditional Spaces}

We now turn to the notion of a conditional space: a structured space that is determined by the state of another space. The interest is in learning and reasoning with distributions over conditional spaces. This is a fundamental notion that arises in the context of causal probabilistic models, which require such conditional distributions when specifying probabilistic relationships between causes and effects. More generally, it arises in directed probabilistic graphical models, in which the graph specifies conditional independence relationships even though it may not have a causal interpretation.

A concrete example comes from the notion of a hierarchical map, which was introduced to better scale the compilation of maps into tractable circuits $[14,79]$. Figure 18 depicts an example of a threelevel hierarchical map with a number of regions. It is a simplified map of neighborhoods in the Los Angeles Westside, where edges represent streets and nodes represent intersections. Nodes of the LA Westside have been partitioned into four sub-regions: Santa Monica, Westwood, Venice and Culver City. Westwood is further partitioned into two sub-regions: UCLA and Westwood Village.

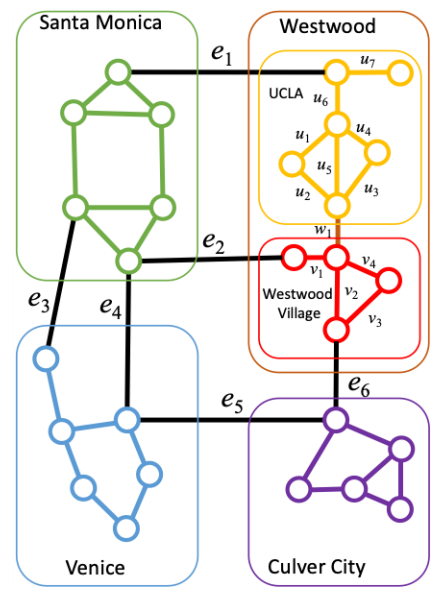

Figure 18: A three-level hierarchical map.

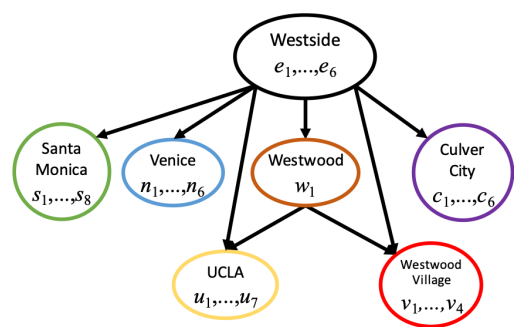

Figure 19: Specifying conditional independence relationships using a directed acyclic graph. The relationship between a node and its parent requires a conditional space.

The main intuition behind a hierarchical map is that navigation behavior in a region $R$ can become independent of navigation behavior in other regions, once we know how region $R$ was entered and exited. These independence relations are specified using a directed acyclic graph as shown in Figure 19 (called a cluster DAG in [78]). Consider the root node 'Westside' which contains variables $e_{1}, \ldots, e_{6}$. These variables represent the roads used to cross between the four (immediate) sub-regions of the Westside. Once we know the state of these variables, we also know how each of these regions may have been entered and exited so their inner navigation behaviors become independent of one another.

Let us now consider Figure 20 to see how the notion of a conditional space arises in this context. The figure highlights Culver City with streets $e_{1}, \ldots, e_{6}$ : the ones for crossing between regions in the Westside. What we need is a structured space over inner roads $c_{1}, \ldots, c_{6}$ of Culver City that specifies valid routes inside the city. But this structured space depends on how we enter and exit the city, which is specified by another space over crossings $e_{1}, \ldots, e_{6}$. That is, the structured space over Culver City roads is conditional on the space over Westside crossings.

The left of Figure 20 expands this example by illustrating the structured space over $c_{1}, \ldots, c_{6}$ assuming we entered/existed Culver city using crossings $e_{3}$ and $e_{4}$ (highlighted in red). The illustration shows some variable assignments that belong to this structured 

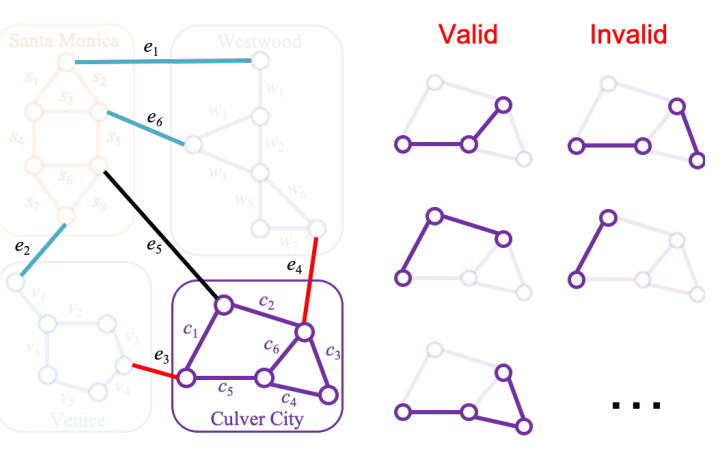

Figure 20: Illustrating the notion of a conditional space. The valid routes inside Culver City are a function of the Westside crossings we use to enter and exit the city.

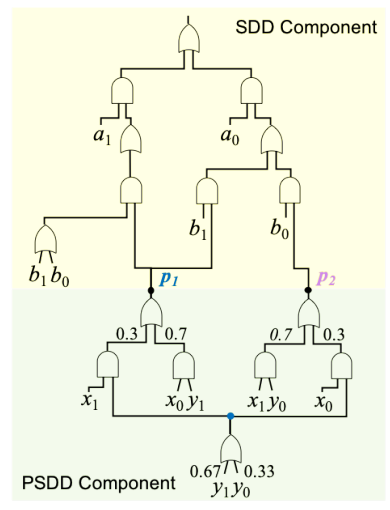

\begin{tabular}{cccc|c}
$A$ & $B$ & $X$ & $Y$ & $P r(X, Y \mid A, B)$ \\
\hline$a_{0}$ & $b_{0}$ & $x_{0}$ & $y_{0}$ & 0.1 \\
$a_{0}$ & $b_{0}$ & $x_{0}$ & $y_{1}$ & 0.2 \\
$a_{0}$ & $b_{0}$ & $x_{1}$ & $y_{0}$ & 0.7 \\
$a_{0}$ & $b_{0}$ & $x_{1}$ & $y_{1}$ & $\mathbf{0 . 0}$ \\
\hline$a_{0}$ & $b_{1}$ & $x_{0}$ & $y_{0}$ & $\mathbf{0 . 0}$ \\
$a_{0}$ & $b_{1}$ & $x_{0}$ & $y_{1}$ & 0.7 \\
$a_{0}$ & $b_{1}$ & $x_{1}$ & $y_{0}$ & 0.1 \\
$a_{0}$ & $b_{1}$ & $x_{1}$ & $y_{1}$ & 0.2 \\
\hline$a_{1}$ & $b_{0}$ & $x_{0}$ & $y_{0}$ & $\mathbf{0 . 0}$ \\
$a_{1}$ & $b_{0}$ & $x_{0}$ & $y_{1}$ & 0.7 \\
$a_{1}$ & $b_{0}$ & $x_{1}$ & $y_{0}$ & 0.1 \\
$a_{1}$ & $b_{0}$ & $x_{1}$ & $y_{1}$ & 0.2 \\
\hline$a_{1}$ & $b_{1}$ & $x_{0}$ & $y_{0}$ & $\mathbf{0 . 0}$ \\
$a_{1}$ & $b_{1}$ & $x_{0}$ & $y_{1}$ & 0.7 \\
$a_{1}$ & $b_{1}$ & $x_{1}$ & $y_{0}$ & 0.1 \\
$a_{1}$ & $b_{1}$ & $x_{1}$ & $y_{1}$ & 0.2 \\
\hline
\end{tabular}

Figure 21: A conditional PSDD and the two conditional distributions it represents.

space (valid) and some that do not (invalid). If we were to enter/exit Culver city using, say, crossings $e_{3}$ and $e_{5}$, then the structured space over $c_{1}, \ldots, c_{6}$ would be different.

Figure 21 depicts a new class of tractable circuits, called conditional PSDDs, which can be used to induce distributions on conditional spaces [78]. In this example, we have a structured space over variables $X, Y$ that is conditioned on a space over variables $A, B$. The conditional PSDD has two components: an SDD circuit (highlighted in yellow) and a multi-rooted PSDD (highlighted in green). The conditional distributions specified by this conditional PSDD are shown on the right of Figure 21. There are two of them: one for state $a_{0}, b_{0}$ of variables $A, B$ and another for the remaining states. The structured space of the first distribution corresponds to the Boolean formula $x_{0} \vee y_{0}$. The structured space for the second distribution corresponds to $x_{1} \vee y_{1}$.

The semantics of a conditional PSDD is relatively simple and illustrated in Figure 24. Consider state $a_{0}, b_{0}$ of variables $A, B$ (right of Figure 24). Evaluating the SDD component at this input leads to selecting the PSDD rooted at $p_{2}$, which generates the distribution conditioned on this state. Evaluating the SDD at any other state of variables $A, B$ leads to selecting the PSDD rooted at $p_{1}$, which generates the distribution for these states (left of Figure 24).

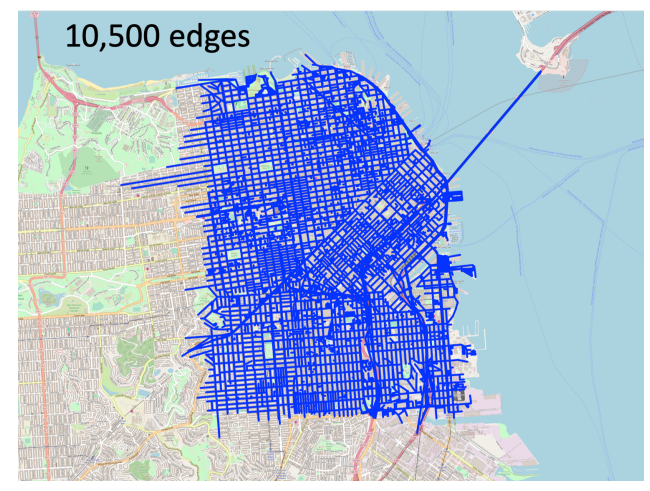

Figure 22: A map of downtown San Fransisco that was compiled into SDD/PSDD circuits.

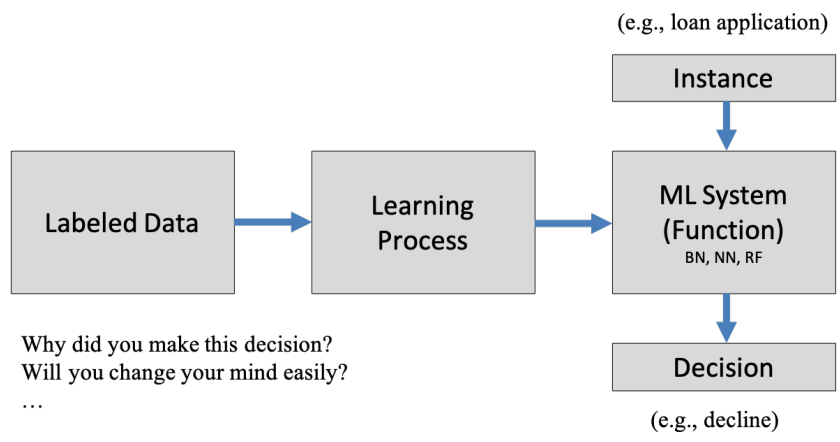

Figure 23: Reasoning about machine learning systems.

When a cluster DAG such as the one in Figure 19 is quantified using conditional PSDDs, the result is known as a structured Bayesian network (SBN) [78]. The conditional PSDDs of an SBN can be multiplied together to yield a classical PSDD over network variables [76]. To give a sense of practical complexity, the map of San Francisco depicted in Figure 22 has 10, 500 edges. A corresponding hierarchical map used in [79] was compiled into a PSDD with size of about 8.9M (edges). The parameters of this SDD were learned from routes collected from GPS data and the induced distribution was used for several reasoning and classification tasks [79]. ${ }^{16}$

\section{LOGIC FOR META REASONING}

We now turn to a most recent role for logic in AI: Reasoning about the behavior of machine learning systems.

Consider Figure 23 which depicts how most machine learning systems are constructed today. We have a labeled dataset that is used to learn a classifier, which is commonly a neural network, a Bayesian network classifier or a random forest. These classifiers are effectively functions that map instances to decisions. For example, an instance could be a loan application and the decision is whether to approve or decline the loan. There is now considerable interest in reasoning about the behavior of such systems. Explaining decisions

\footnotetext{
${ }^{16}$ See https://github.com/hahaXD/hierarchical_map_compiler for a package that compiles route constraints into an SDD.
} 


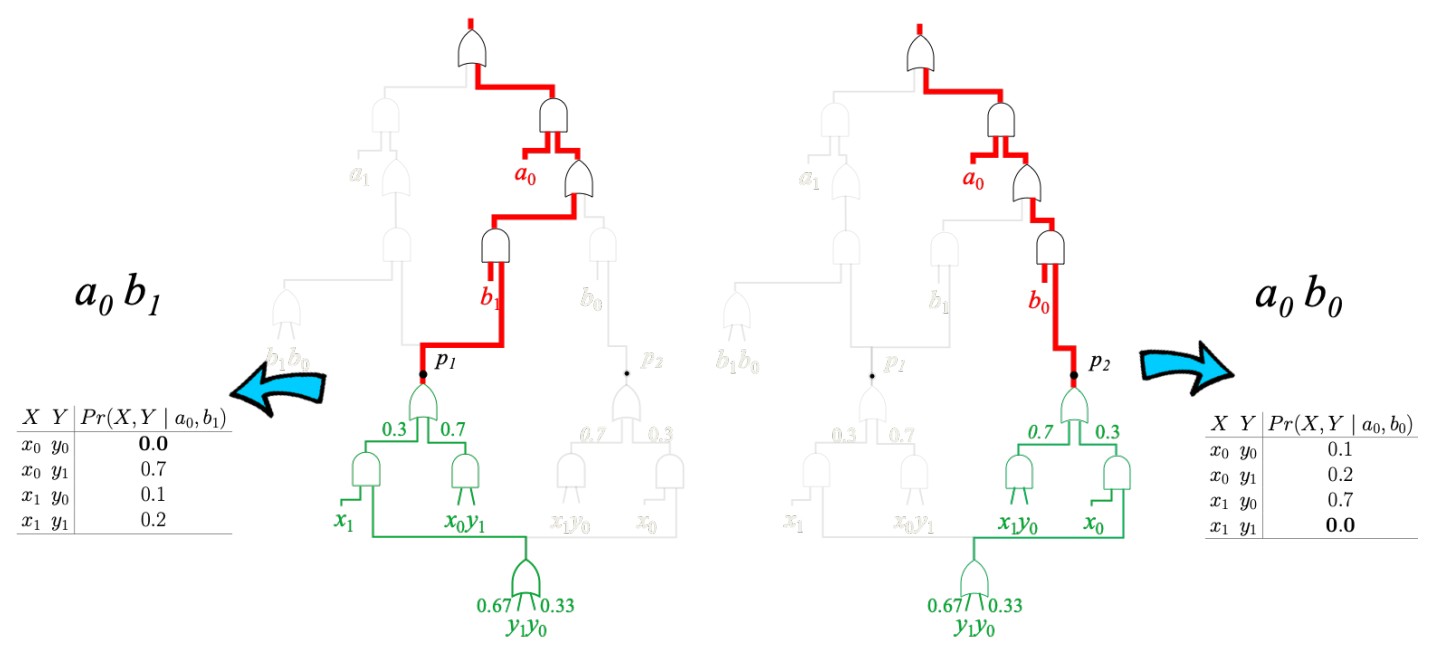

Figure 24: Selecting conditional distributions using the conditional PSDD of Figure 21.

is at the forefront of current interests: Why did you decline Maya's application? Quantifying the robustness of these decisions is also attracting a lot of attention: Would reversing the decision on Maya require many changes to her application? In some domains, one expects the learned systems to satisfy certain properties, like monotonicity, and there is again an interest in proving such properties formally. For example, can we guarantee that a loan applicant will be approved when the only difference they have with another approved applicant is their higher income? These interests, however, are challenged by the numeric nature of machine learning systems and the fact that these systems are often model-free, e.g., neural networks, so they appear as black boxes that are hard to analyze.

The third role for logic we discuss next rests on the following observation: Even though these machine learning classifiers are learned from data and numeric in nature, they often implement discrete decision functions. One can therefore extract these decisions functions and represent them symbolically using tractable circuits. The outcome of this process is a circuit that precisely captures the input-output behavior of the machine learning system, which can then be used to reason about its behavior. This includes explaining decisions, measuring robustness and formally proving properties.

Consider the example in Figure 25 which pertains to one of the simplest machine learning systems: a Naive Bayes classifier. We have class variable $P$ and three features $B, U$ and $S$. Given an instance (patient) and their test results $b, u$ and $s$, this classifier renders a decision by computing the posterior probability $\operatorname{Pr}(p \mid b, u, s)$ and then checking whether it passes a given threshold $T$. If it does, we declare a positive decision; otherwise, a negative decision.

While this classifier is numeric and its decisions are based on probabilistic reasoning, it does induce a discrete decision function. In fact, the function is Boolean in this case as it maps the Boolean variables $B, U$ and $S$, which correspond to test results, into a binary decision. This observation was originally made in [9], which proposed the compilation of Naive Bayes classifiers into symbolic

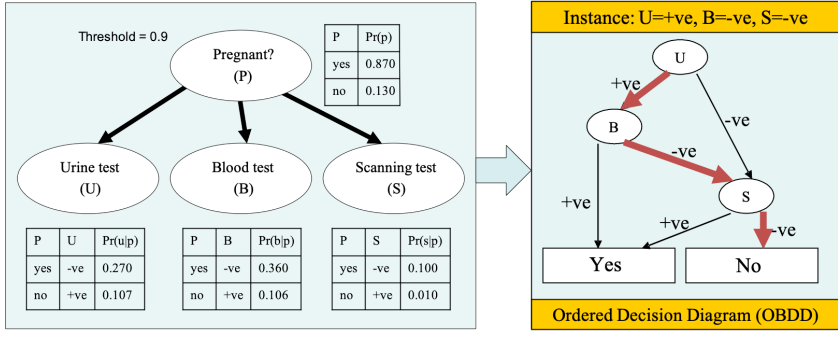

Figure 25: Compiling a Naive Bayes classifier into a symbolic decision graph (a tractable NNF circuit).

decision graphs as shown in Figure 25. For every instance, the decision made by the (probabilistic) naive Bayes classifier is guaranteed to be the same as the one made by the (symbolic) decision graph.

The compilation algorithm of [9] generates Ordered Decision Diagrams (ODDs), which correspond to Ordered Binary Decision Diagrams (OBDDs) when the features are binary. Recall from Figure 11 that an OBDD corresponds to a tractable NNF circuit (once one adjusts for notation). Hence, the proposal in [9] amounted to compiling a Naive Bayes classifier into a tractable NNF circuit that precisely captures its input-output behavior. This compilation algorithm was recently extended to Bayesian network classifiers with tree structures [82] and later to Bayesian network classifiers with arbitrary structures [83]. ${ }^{17}$ Certain classes of neural networks can also be compiled into tractable circuits, which include SDD circuits as shown in $[15,80,84]$.

While Bayesian and neural networks are numeric in nature, random forests are not (at least the ones with majority voting). Hence, random forests represent less of a challenge for this role of logic as we can easily encode the input-output behavior of a random forest using a Boolean formula. We first encode each decision tree into a Boolean formula, which is straightforward even in the presence of

\footnotetext{
${ }^{17}$ See http://reasoning.cs.ucla.edu/xai/ for related software.
} 
continuous variables (the learning algorithm discretizes the variables). We then combine these formulas using a majority circuit. The remaining challenge is purely computational as we now need to compile the Boolean formula into a suitable tractable circuit.

We next turn to reasoning about the behavior of classifiers, assuming they have been compiled into tractable circuits.

\subsection{Explaining Decisions}

Consider the classifier in Figure 25 and Susan who tested positive for the blood, urine and scanning tests. The classifier says that Susan is pregnant and we need to know why.

The first notion to address this question is the PI-explanation introduced in [82] and termed sufficient reason in [33] (to make distinctions with other types of reasons). A sufficient reason is a minimal set of instance characteristics that is guaranteed to trigger the decision, regardless of what the other characteristics might be. In this example, Susan would be classified as pregnant as long as she tests positive for the scanning test; that is, regardless of what the other two test results are. Hence, $S=+v e$ is a sufficient reason for the decision. There is only one other sufficient reason for this decision: $B=+v e, U=+v e$. Combining the two sufficient reasons we get $S=+v e \vee(B=+v e, U=+v e)$, which is called the complete reason behind the decision [33] or simply the decision's reason.

The reason behind a decision provides the most general abstraction of an instance that can trigger the decision. Any instance property that can trigger the decision is captured by the reason. The reason behind a decision can also be used to decide whether the decision is biased, and in some cases whether the classifier itself is biased even when the considered decision is not. We will provide concrete examples later but we first need to establish the semantics of sufficient and complete reasons.

\begin{tabular}{l|l|} 
Boolean function: $f=(A+\bar{C})(B+C)(A+B)$ & $\begin{array}{l}\text { Boolean function: } \bar{f}=\overline{(A+\bar{C})(B+C)(A+B)} \\
\text { Prime implicants: } A B, A C, B \bar{C}\end{array}$ \\
& Prime implicants: $\bar{A} C, \bar{B} \bar{C}, \bar{A} \bar{B}$ \\
Instance: $A B \bar{C}$ & Instance: $\bar{A} B C$ \\
Decision: 1 & Decision: 0 \\
Sufficient reasons: $A B, B \bar{C}$ & Sufficient reasons: $\bar{A} C$
\end{tabular}

\section{Figure 26: Prime implicants of Boolean functions.}

These notions are based on prime implicants of Boolean functions, which have been studied extensively in the literature $[21,53,69,70]$. Consider the Boolean function $f$ in Figure 26 over variables $A, B$ and $C$. A prime implicant of the function is a minimal setting of its variables that causes the function to trigger. This function has three prime implicants as shown in the figure: $A B, A C$ and $B \bar{C}$. Consider now the instance $A B \bar{C}$ leading to a positive decision $f(A B \bar{C})=1$. The sufficient reasons for this decision are the prime implicants of function $f$ that are compatible with the instance: $A B$ and $B \bar{C}$. Explaining negative decisions requires working with the function's complement $\bar{f}$. Consider instance $\bar{A} B C$, which sets the function to 0 . The complement $\bar{f}$ has three prime implicants $\bar{A} C, \bar{B} \bar{C}$ and $\bar{A} \bar{B}$. Only one of these is compatible with the instance, $\bar{A} C$, so it is the only sufficient reason for the decision on this instance. ${ }^{18}$

\footnotetext{
${ }^{18}$ The popular Anchor [71] system can be viewed as computing approximations of sufficient reasons. The quality of these approximations has been evaluated on some
}

Sufficient reasons can be used to reason about decision and classifier bias, which are defined in the context of protected features. A decision on an instance is biased iff it would be different had we only changed protected features in the instance. A classifier is biased iff it makes at least one biased decision. If every sufficient reason of a decision contains at least one protected feature, the decision is guaranteed to be biased [33]. If some but not all sufficient reasons contain protected features, the decision is not biased but the classifier is guaranteed to be biased (that is, the classifier will make a biased decision on some other instance).

Consider Figure 27 which depicts an admissions classifier in the form of an OBDD (the classifier could have been compiled from a Bayesian network, a neural network or a random forest). The classifier has five features, one of them is protected: whether the applicant comes from a rich hometown $(R)$. Robin is admitted by the classifier and the decision has five sufficient reasons, depicted in Figure 27. Three of these sufficient reasons contain the protected feature $R$ and two do not. Hence, the decision on Robin is not biased, but the classifier is biased. Consider now Scott who is also admitted. The decision on Scott has four sufficient reasons and all of them contain a protected feature. Hence, the decision on Scott is biased: it will be reversed if Scott were not to come from a rich hometown.

A decision may have an exponential number of reasons, which makes it impractical to analyze decisions by enumerating sufficient reasons. One can use the complete reason behind a decision for this purpose as it contains all the needed information. Moreover, if the classifier is represented by an appropriate tractable circuit, then the complete reason behind a decision can be extracted from the classifier in linear time, in the form of another tractable circuit called the reason circuit [33]. Figure 27 depicts the reason circuits for decisions on Robin and Scott. Reason circuits get their tractability from being monotone, allowing one to efficiently reason about decisions including their bias. One can also reason about counterfactuals once the reason circuit for a decision is constructed. For example, one can efficiently evaluate statements such as: The decision on April would stick even if she were not to have work experience because she passed the entrance exam; see [33] for details.

We conclude this section by pointing to an example from [80], which compiled a Convolutional Neural Network (CNN) that classifies digits 0 and 1 in $16 \times 16$ images. The $\mathrm{CNN}$ had an accuracy of $98.74 \%$. Figure 28 depicts an image that was classified correctly as containing digit 0 . One of the sufficient reasons for this decision is also shown in the figure, which includes only 3 pixels out of 256 . If these three pixels are kept white, the CNN will classify the image as containing digit 0 regardless of the state of other pixels.

\subsection{Robustness and Formal Properties}

One can define both decision and model robustness. The robustness of a decision is defined as the smallest number of features that need to flip before the decision flips [81]. Model robustness is defined as

\footnotetext{
datasets and corresponding classifiers in [41], where an approximation is called optimistic if it is a strict subset of a sufficient reason and pessimistic if it is a strict superset of a sufficient reason. Anchor computes approximate explanations without having to abstract the machine learning system into a symbolic representation. Another set of approaches abstract the behavior into symbolic form and compute sufficient reasons or other verification queries exactly, but using SAT-based techniques instead of compiling into tractable circuits; see, e.g., [39, 40, 43, 48, 58, 82, 84].
} 

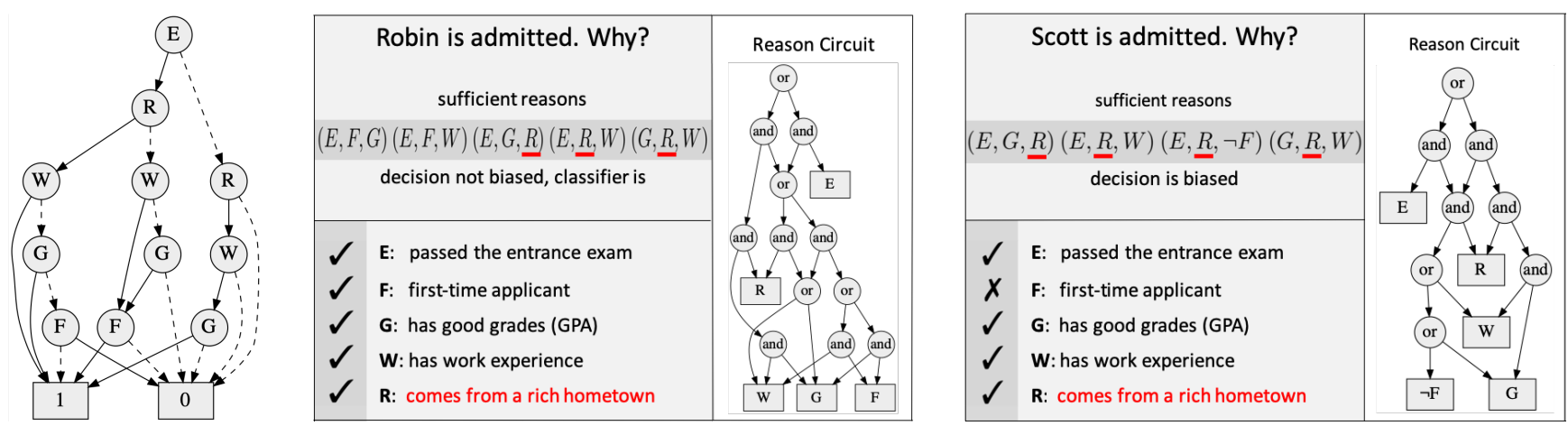

Figure 27: Explaining admission decisions of an OBDD classifier (left). The reason circuit represents the complete reason behind a decision (a disjunction of all its sufficient reasons). The reason circuit is monotone and hence tractable.

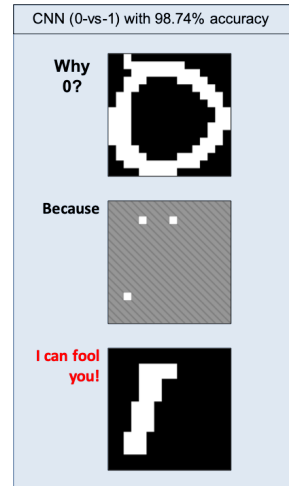

Figure 28: Explaining the decisions of a neural network.

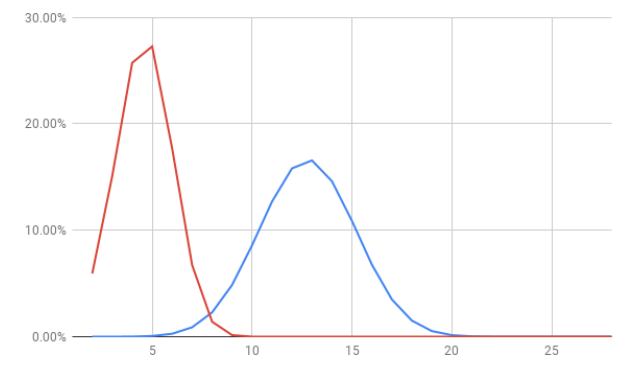

Figure 29: Robustness level vs. proportion of instances for two neural networks with similar accuracies. Net 1 is plotted in blue (right) and Net 2 in red (left).

the average decision robustness (over all possible instances) [80]. Decision robustness is coNP-complete and model robustness is \#Phard. If the decision function is represented using a tractable circuit of a suitable type, which includes OBDDs, then decision robustness can be computed in time linear in the circuit size [81]. Model robustness can be computed using a sequence of polytime operations but the total complexity is not guaranteed to be in polytime [80].

Figure 29 depicts an example of robustness analysis for two CNNs that classify digits 1 and 2 in $16 \times 16$ images [80]. The two CNNs have the same architectures but were trained using two different parameter seeds, leading to testing accuracies of 98.18 (Net 1) and 96.93 (Net 2). The CNNs were compiled into SDD circuits where the SDD of Net 1 had 3, 653 edges and the one for Net 2 had only 440 edges. The two CNNs are similar in terms of accuracy (differing by only $1.25 \%$ ) but are very different when compared by robustness. For example, Net 1 attained a model robustness of 11.77 but Net 2 obtained a robustness of only 3.62. For Net 2, this means that on average, 3.62 pixel flips are needed to flip a digit-1 classification to digit-2, or vice versa. Moreover, the maximum robustness of Net 1 was 27, while that of Net 2 was only 13 . For Net 1, this means that there is an instance that would not flip classification unless one is allowed to flip at least 27 of its pixels. For Net 2, it means the decision on any instance can be flipped if one is allowed to flip 13 or more pixels. Note that Figure 29 reports the robustness of $2^{256}$ instances for each $\mathrm{CNN}$, which is made possible by having captured the input-output behavior of these CNNs using tractable circuits.

In the process of compiling a neural network into a tractable circuit as proposed in $[15,80]$, one also compiles each neuron into its own tractable circuit. This allows one to interpret the functionality of each neuron by analyzing the corresponding tractable circuit, which is a function of the network's inputs. For example, if the tractable circuit supports model counting in polytime, then one can efficiently answer questions such as: Of all network inputs that cause a neuron to fire, what proportion of them set input $X_{i}$ to 1 ? This is just one mode of analysis that can be performed efficiently once the input-output behavior of a machine learning system is compiled into a suitable tractable circuit. Other examples include monotonicity analysis, which is discussed in [81].

\section{CONCLUSION AND OUTLOOK}

We reviewed three modern roles for logic in artificial intelligence: logic as a basis for computation, logic for learning from a combination of data and knowledge, and logic for reasoning about the behavior of machine learning systems.

Everything we discussed was contained within the realm of propositional logic and based on the theory of tractable Boolean circuits. The essence of these roles is based on an ability to compile Boolean formula into Boolean circuits with appropriate properties. As such, the bottleneck for advancing these roles further-at least 
as far as enabling more scalable applications-boils down to the need for improving the effectiveness of knowledge compilers (i.e., algorithms that enforce certain properties on NNF circuits). The current work on identifying (more) tractable circuits and further studying their properties appears to be ahead of work on improving compilation algorithms. This imbalance needs to be addressed, perhaps by encouraging more open source releases of knowledge compilers and the nurturing of regular competitions as has been done successfully by the SAT community. ${ }^{19}$

McCarthy's proposal for using logic as the basis for knowledge representation and reasoning had an overwhelming impact [52] But it also entrenched into our thinking a role for logic that has now been surpassed, while continuing to be the dominant role associated with logic in textbooks on artificial intelligence. Given the brittleness of purely symbolic representations in key applications, and the dominance today of learned, numeric representations, this entrenched association has pushed logic to a lower priority in artificial intelligence research than what can be rationally justified.

Another major (and more recent) transition relates to the emergence of machine learning "boxes," which have expanded the scope and utility of symbolic representations and reasoning. While symbolic representations may be too brittle to fully represent certain aspects of the real world, they are provably sufficient for representing the behavior of certain machine learning boxes (and exactly when the box's inputs/outputs are discrete). This has created a new role for logic in "reasoning about what was learned" in contrast to the older and entrenched role of reasoning about the real world.

The latter transition is more significant than may appear on first sight. For example, the behavior of a machine learning box is driven by the box's internal causal mechanisms and can therefore be subjected to causal reasoning and analysis-even when the box itself was built using ad hoc techniques and may have therefore missed on capturing causality of the real world. Machine learning boxes, called "The AI" by many today, are now additional inhabitants of the real world. As such, they should be viewed as a new "subject" of logical and causal reasoning perhaps more so than their competitor.

These modern transitions-and the implied new modes of interplay between logic, probabilistic reasoning and machine learningneed to be paralleled by a transition in AI education that breaks away from the older models of viewing these areas as being either in competition or merely in modular harmony. What we need here is not only integration of these methods but also their fusion. Quoting [30]: "We need a new generation of AI researchers who are well versed in and appreciate classical AI, machine learning, and computer science more broadly while also being informed about AI history." Cultivating such a generation of AI researchers is another bottleneck to further advance the modern roles of logic in $\mathrm{AI}$ and to further advance the field of $\mathrm{AI}$ as a whole.

\section{ACKNOWLEDGMENTS}

I wish to thank Arthur Choi and Jason Shen for their valuable feedback and help with some of the figures. This work has been partially supported by grants from NSF IIS-1910317, ONR N0001418-1-2561, DARPA N66001-17-2-4032 and a gift from JP Morgan.

\footnotetext{
${ }^{19}$ www.satlive.org
}

\section{REFERENCES}

[1] Antoine Amarilli. 2019. Provenance in Databases and Links to Knowledge Compilation. In KOCOON workshop on knowledge compilation. http://kocoon.gforge. inria.fr/slides/amarilli.pdf.

[2] Paul Beame and Vincent Liew. 2015. New Limits for Knowledge Compilation and Applications to Exact Model Counting. In UAI. AUAI Press, 131-140.

[3] Beate Bollig and Matthias Buttkus. 2019. On the Relative Succinctness of Sentential Decision Diagrams. Theory Comput. Syst. 63, 6 (2019), 1250-1277.

[4] Craig Boutilier, Nir Friedman, Moisés Goldszmidt, and Daphne Koller. 1996. Context-Specific Independence in Bayesian Networks. In UAI. 115-123.

[5] Simone Bova. 2016. SDDs Are Exponentially More Succinct than OBDDs. In AAAI. 929-935.

[6] Simone Bova, Florent Capelli, Stefan Mengel, and Friedrich Slivovsky. 2016. Knowledge Compilation Meets Communication Complexity. In IfCAI. IJCAI/AAAI Press, 1008-1014.

[7] Randal E. Bryant. 1986. Graph-Based Algorithms for Boolean Function Manipulation. IEEE Trans. Computers 35, 8 (1986), 677-691.

[8] Marco Cadoli and Francesco M. Donini. 1997. A Survey on Knowledge Compilation. AI Commun. 10, 3,4 (1997), 137-150.

[9] Hei Chan and Adnan Darwiche. 2003. Reasoning About Bayesian Network Classifiers. In Proceedings of the Nineteenth Conference on Uncertainty in Artificial Intelligence (UAI). 107-115.

[10] Mark Chavira and Adnan Darwiche. 2008. On Probabilistic Inference by Weighted Model Counting. Artificial Intelligence 172, 6-7 (April 2008), 772-799.

[11] Suming Jeremiah Chen, Arthur Choi, and Adnan Darwiche. 2015. Value of Information Based on Decision Robustness. In AAAI. AAAI Press, 3503-3510.

[12] Arthur Choi and Adnan Darwiche. 2013. Dynamic Minimization of Sentential Decision Diagrams. In Proceedings of the 27th Conference on Artificial Intelligence (AAAI).

[13] Arthur Choi and Adnan Darwiche. 2017. On Relaxing Determinism in Arithmetic Circuits. In Proceedings of the Thirty-Fourth International Conference on Machine Learning (ICML). 825-833.

[14] Arthur Choi, Yujia Shen, and Adnan Darwiche. 2017. Tractability in Structured Probability Spaces. In NIPS.

[15] Arthur Choi, Weijia Shi, Andy Shih, and Adnan Darwiche. 2019. Compiling Neural Networks into Tractable Boolean Circuits. In AAAI Spring Symposium on Verification of Neural Networks (VNN).

[16] Arthur Choi, Nazgol Tavabi, and Adnan Darwiche. 2016. Structured Features in Naive Bayes Classification. In AAAI.

[17] Arthur Choi, Guy Van den Broeck, and Adnan Darwiche. 2015. Tractable Learning for Structured Probability Spaces: A Case Study in Learning Preference Distributions. In IFCAI.

[18] Arthur Choi, Yexiang Xue, and Adnan Darwiche. 2012. Same-Decision Probability: A Confidence Measure for Threshold-Based Decisions. International fournal of Approximate Reasoning (IfAR) 53, 9 (2012), 1415-1428.

[19] YooJung Choi, Adnan Darwiche, and Guy Van den Broeck. 2017. Optimal Feature Selection for Decision Robustness in Bayesian Networks. In IfCAI. ijcai.org, $1554-1560$.

[20] YooJung Choi and Guy Van den Broeck. 2018. On Robust Trimming of Bayesian Network Classifiers. In IfCAI. ijcai.org, 5002-5009.

[21] Yves Crama and Peter L. Hammer. 2011. Boolean Functions - Theory, Algorithms, and Applications. Encyclopedia of mathematics and its applications, Vol. 142. Cambridge University Press.

[22] Adnan Darwiche. 2001. Decomposable Negation Normal Form. F. ACM 48, 4 (2001), 608-647.

[23] Adnan Darwiche. 2001. On the Tractable Counting of Theory Models and its Application to Truth Maintenance and Belief Revision. Fournal of Applied NonClassical Logics 11, 1-2 (2001), 11-34.

[24] Adnan Darwiche. 2002. A Logical Approach to Factoring Belief Networks. In KR. 409-420.

[25] Adnan Darwiche. 2003. A Differential Approach to Inference in Bayesian Networks. FACM 50, 3 (2003), 280-305.

[26] Adnan Darwiche. 2004. New Advances in Compiling CNF into Decomposable Negation Normal Form. In ECAI. 328-332.

[27] Adnan Darwiche. 2009. Modeling and Reasoning with Bayesian Networks. Cambridge University Press.

[28] Adnan Darwiche. 2011. SDD: A New Canonical Representation of Propositional Knowledge Bases. In IfCAI. 819-826.

[29] Adnan Darwiche. 2014. Tractable Knowledge Representation Formalisms. In Tractability, Lucas Bordeaux, Youssef Hamadi, and Pushmeet Kohli (Eds.). Cambridge University Press, 141-172.

[30] Adnan Darwiche. 2018. Human-level intelligence or animal-like abilities? Commun. ACM 61, 10 (2018), 56-67.

[31] Adnan Darwiche and Arthur Choi. 2010. Same-Decision Probability: A Confidence Measure for Threshold-Based Decisions under Noisy Sensors. In Proceedings of the Fifth European Workshop on Probabilistic Graphical Models (PGM). $113-120$. 
[32] Adnan Darwiche, Rina Dechter, Arthur Choi, Vibhav Gogate, and Lars Otten. 2008. Results from the Probabilistic Inference Evaluation of UAI-08. (2008).

[33] Adnan Darwiche and Auguste Hirth. 2020. On The Reasons Behind Decisions. In Proceedings of the 24th European Conference on Artificial Intelligence (ECAI).

[34] Adnan Darwiche and Pierre Marquis. 2002. A knowledge compilation map. JAIR 17 (2002), 229-264.

[35] Anton Dries, Angelika Kimmig, Wannes Meert, Joris Renkens, Guy Van den Broeck, Jonas Vlasselaer, and Luc De Raedt. 2015. ProbLog2: Probabilistic Logic Programming. In ECML/PKDD (3) (Lecture Notes in Computer Science), Vol. 9286. Springer, 312-315.

[36] Michael A Fligner and Joseph S Verducci. 1986. Distance based ranking models Journal of the Royal Statistical Society. Series B (Methodological) (1986), 359-369.

[37] Jinbo Huang, Mark Chavira, and Adnan Darwiche. 2006. Solving MAP Exactly by Searching on Compiled Arithmetic Circuits. In AAAI. 1143-1148.

[38] Jinbo Huang and Adnan Darwiche. 2007. The Language of Search. 7. Artif. Intell. Res. (FAIR) 29 (2007), 191-219.

[39] Alexey Ignatiev, Nina Narodytska, and João Marques-Silva. 2019. AbductionBased Explanations for Machine Learning Models. In Proceedings of the ThirtyThird Conference on Artificial Intelligence (AAAI). 1511-1519.

[40] Alexey Ignatiev, Nina Narodytska, and João Marques-Silva. 2019. On Relating Explanations and Adversarial Examples. In Advances in Neural Information Processing Systems 32 (NeurIPS). 15857-15867.

[41] Alexey Ignatiev, Nina Narodytska, and João Marques-Silva. 2019. On Validating, Repairing and Refining Heuristic ML Explanations. CoRR abs/1907.02509 (2019).

[42] Abhay Kumar Jha and Dan Suciu. 2013. Knowledge Compilation Meets Database Theory: Compiling Queries to Decision Diagrams. Theory Comput. Syst. 52, 3 (2013), 403-440

[43] Guy Katz, Clark W. Barrett, David L. Dill, Kyle Julian, and Mykel J. Kochenderfer 2017. Reluplex: An Efficient SMT Solver for Verifying Deep Neural Networks. In Computer Aided Verification CAV. 97-117.

[44] Doga Kisa, Guy Van den Broeck, Arthur Choi, and Adnan Darwiche. 2014. Probabilistic Sentential Decision Diagrams. In $K R$.

[45] D. Koller and N. Friedman. 2009. Probabilistic Graphical Models: Principles and Techniques. MIT Press.

[46] Jean-Marie Lagniez and Pierre Marquis. 2017. An Improved Decision-DNNF Compiler. In IfCAI. ijcai.org, 667-673.

[47] Anna L. D. Latour, Behrouz Babaki, Anton Dries, Angelika Kimmig, Guy Van den Broeck, and Siegfried Nijssen. 2017. Combining Stochastic Constraint Optimization and Probabilistic Programming - From Knowledge Compilation to Constraint Solving. In CP (Lecture Notes in Computer Science), Vol. 10416. Springer, 495-511.

[48] Francesco Leofante, Nina Narodytska, Luca Pulina, and Armando Tacchella 2018. Automated Verification of Neural Networks: Advances, Challenges and Perspectives. CoRR abs/1805.09938 (2018).

[49] Colin L. Mallows. 1957. Non-null ranking models. Biometrika (1957).

[50] Robin Manhaeve, Sebastijan Dumancic, Angelika Kimmig, Thomas Demeester and Luc De Raedt. 2018. DeepProbLog: Neural Probabilistic Logic Programming. In Advances in Neural Information Processing Systems 31, S. Bengio, H. Wallach, H. Larochelle, K. Grauman, N. Cesa-Bianchi, and R. Garnett (Eds.). Curran Associates, Inc., 3749-3759. http://papers.nips.cc/paper/7632-deepproblog-neuralprobabilistic-logic-programming.pdf

[51] Pierre Marquis. 1995. Knowledge Compilation Using Theory Prime Implicates. In IfCAI. 837-845.

[52] John McCarthy. 1959. Programs with common sense. In Proceedings of the Teddington Conference on the Mechanization of Thought Processes. http://wwwformal.stanford.edu/jmc/mcc59.html

[53] E. J. McCluskey. 1956. Minimization of Boolean functions. The Bell System Technical fournal 35, 6 (Nov 1956), 1417-1444. https://doi.org/10.1002/j.15387305.1956.tb03835.x

[54] Marina Meila and Harr Chen. 2010. Dirichlet process mixtures of generalized Mallows models. In Proceedings of UAI.

[55] Christoph Meinel and Thorsten Theobald. 1998. Algorithms and Data Structures in VLSI Design: OBDD - Foundations and Applications. Springer.

[56] Christian J. Muise, Sheila A. McIlraith, J. Christopher Beck, and Eric I. Hsu. 2012 Dsharp: Fast d-DNNF Compilation with sharpSAT. In Canadian Conference on AI (Lecture Notes in Computer Science), Vol. 7310. Springer, 356-361.

[57] Kevin Patrick Murphy. 2012. Machine Learning: A Probabilistic Perspective. MIT Press.

[58] Nina Narodytska, Shiva Prasad Kasiviswanathan, Leonid Ryzhyk, Mooly Sagiv, and Toby Walsh. 2018. Verifying Properties of Binarized Deep Neural Networks. In Proceedings of the Thirty-Second AAAI Conference on Artificial Intelligence $(A A A I)$.

[59] N.J. Nilsson. 1986. Probabilistic logic. Artificial intelligence 28, 1 (1986), 71-87.

[60] Masaaki Nishino, Norihito Yasuda, Shin-ichi Minato, and Masaaki Nagata. 2017. Compiling Graph Substructures into Sentential Decision Diagrams. In AAAI. 1213-1221.

[61] Umut Oztok, Arthur Choi, and Adnan Darwiche. 2016. Solving $\mathrm{PP}^{\mathrm{PP}}$-Complete Problems Using Knowledge Compilation. In Proceedings of the 15th International Conference on Principles of Knowledge Representation and Reasoning (KR). 94-103.
[62] Umut Oztok and Adnan Darwiche. 2014. On Compiling CNF into Decision-DNNF. In $C P .42-57$.

[63] Umut Oztok and Adnan Darwiche. 2018. An Exhaustive DPLL Algorithm for Model Counting. 7. Artif. Intell. Res. 62 (2018), 1-32.

[64] James D. Park and Adnan Darwiche. 2004. Complexity Results and Approximation Strategies for MAP Explanations. F. Artif. Intell. Res. (FAIR) 21 (2004), 101-133.

[65] J. Pearl. 1988. Probabilistic Reasoning in Intelligent Systems: Networks of Plausible Inference. Morgan Kaufmann.

[66] Knot Pipatsrisawat and Adnan Darwiche. 2008. New Compilation Languages Based on Structured Decomposability. In AAAI. 517-522.

[67] Knot Pipatsrisawat and Adnan Darwiche. 2009. A New d-DNNF-Based Bound Computation Algorithm for Functional EMAJSAT. In IfCAI. 590-595.

[68] Hoifung Poon and Pedro M. Domingos. 2011. Sum-Product Networks: A New Deep Architecture. In UAI. 337-346.

[69] W. V. Quine. 1952. The Problem of Simplifying Truth Functions. The American Mathematical Monthly 59, 8 (1952), 521-531. http://www.jstor.org/stable/2308219

[70] W. V. Quine. 1959. On Cores and Prime Implicants of Truth Functions. The American Mathematical Monthly 66, 9 (1959), 755-760. http://www.jstor.org/ stable/2310460

[71] Marco Túlio Ribeiro, Sameer Singh, and Carlos Guestrin. 2018. Anchors: HighPrecision Model-Agnostic Explanations. In AAAI. AAAI Press, 1527-1535.

[72] Dan Roth. 1996. On the Hardness of Approximate Reasoning. AIf 82, 1-2 (1996), 273-302.

[73] Tian Sang, Paul Beame, and Henry A. Kautz. 2005. Performing Bayesian Inference by Weighted Model Counting. In AAAI. 475-482.

[74] Bart Selman and Henry A. Kautz. 1996. Knowledge Compilation and Theory Approximation. FACM 43, 2 (1996), 193-224.

[75] Shubham Sharma, Rahul Gupta, Subhajit Roy, and Kuldeep S. Meel. 2018. Knowledge Compilation meets Uniform Sampling. In LPAR (EPiC Series in Computing), Vol. 57. EasyChair, 620-636.

[76] Yujia Shen, Arthur Choi, and Adnan Darwiche. 2016. Tractable Operations for Arithmetic Circuits of Probabilistic Models. In Advances in Neural Information Processing Systems 29 (NIPS).

[77] Yujia Shen, Arthur Choi, and Adnan Darwiche. 2017. A Tractable Probabilistic Model for Subset Selection. In Proceedings of the 33rd Conference on Uncertainty in Artificial Intelligence (UAI).

[78] Yujia Shen, Arthur Choi, and Adnan Darwiche. 2018. Conditional PSDDs: Modeling and Learning With Modular Knowledge. In AAAI. AAAI Press, 6433-6442.

[79] Yujia Shen, Anchal Goyanka, Adnan Darwiche, and Arthur Choi. 2019. Structured Bayesian Networks: From Inference to Learning with Routes. In AAAI. AAAI Press, 7957-7965.

[80] Weijia Shi, Andy Shih, Adnan Darwiche, and Arthur Choi. 2020. On Tractable Representations of Binary Neural Networks. http://arxiv.org/abs/2004.02082.

[81] Andy Shih, Arthur Choi, and Adnan Darwiche. 2018. Formal Verification of Bayesian Network Classifiers. In Proceedings of the 9th International Conference on Probabilistic Graphical Models (PGM).

[82] Andy Shih, Arthur Choi, and Adnan Darwiche. 2018. A Symbolic Approach to Explaining Bayesian Network Classifiers. In Proceedings of the 27th International foint Conference on Artificial Intelligence (IFCAI).

[83] Andy Shih, Arthur Choi, and Adnan Darwiche. 2019. Compiling Bayesian Network Classifiers into Decision Graphs. In AAAI. AAAI Press, 7966-7974.

[84] Andy Shih, Adnan Darwiche, and Arthur Choi. 2019. Verifying Binarized Neural Networks by Angluin-Style Learning. In SAT

[85] Andy Shih, Guy Van den Broeck, Paul Beame, and Antoine Amarilli. 2019. Smoothing Structured Decomposable Circuits. In NeurIPS. 11412-11422.

[86] Solomon Eyal Shimony. 1994. Finding MAPs for Belief Networks is NP-Hard. Artif. Intell. 68, 2 (1994), 399-410.

[87] Friedrich Slivovsky. 2019. An Introduction to Knowledge Compilation. In KOCOON workshop on knowledge compilation. http://kocoon.gforge.inria.fr/slides/ slivovsky.pdf.

[88] Marc Thurley. 2006. sharpSAT - Counting Models with Advanced Component Caching and Implicit BCP. In SAT (Lecture Notes in Computer Science), Vol. 4121. Springer, 424-429.

[89] Guy Van den Broeck and Adnan Darwiche. 2015. On the Role of Canonicity in Knowledge Compilation. In $A A A I$

[90] Ingo Wegener. 2000. Branching Programs and Binary Decision Diagrams. SIAM.

[91] Yaqi Xie, Ziwei Xu, Kuldeep S. Meel, Mohan S. Kankanhalli, and Harold Soh. 2019. Embedding Symbolic Knowledge into Deep Networks. In NeurIPS. 4235-4245.

[92] Jingyi Xu, Zilu Zhang, Tal Friedman, Yitao Liang, and Guy Van den Broeck. 2018. A Semantic Loss Function for Deep Learning with Symbolic Knowledge. In ICML (Proceedings of Machine Learning Research), Vol. 80. PMLR, 5498-5507.

[93] Yexiang Xue, Arthur Choi, and Adnan Darwiche. 2012. Basing Decisions on Sentences in Decision Diagrams. In AAAI. 842-849. 